\title{
Localized Level Crossing Random Walk Test Robust to the Presence of Structural Breaks*
}

\author{
Vitali Alexeev ${ }^{\dagger 1,2}$ and Alex Maynard ${ }^{2}$ \\ ${ }^{1}$ School of Economics and Finance, University of Tasmania, Hobart, Tasmania 7001, Australia \\ ${ }^{2}$ Department of Economics, University of Guelph, Guelph, Ontario N1G 2W1, Canada
}

April 28, 2010

\begin{abstract}
We propose a modified version of the nonparametric level crossing random walk test, in which the crossing level is determined locally. This modification results in a test that is robust to unknown multiple structural breaks in the level and slope of the trend function under both the null and alternative hypothesis. No knowledge regarding the number or timing of the breaks is required. An algorithm is proposed to select the degree of localization in order to maximize bootstrapped power in a proximate model. A computational procedure is then developed to adjust the critical values for the effect of this selection procedure by replicating it under the null hypothesis. The test is applied to Canadian nominal inflation and nominal interest rate series with implications for the Fisher hypothesis.
\end{abstract}

Keyword: Level crossing, random walk, structural breaks, unit root, robustness.

\section{Introduction}

Originally examined by Kendall [1953], the random walk hypothesis has been important in numerous disciplines including economics, finance, and international finance, in which random walk models have been used to model variables such as consumption, stock prices, and exchange rates. Likewise, tests of the random walk hypothesis are frequently employed to test deeper theoretical models, such as the permanent income hypothesis and weak form market efficiency. Tests of weak form market efficiency include serial correlation tests, runs tests, and the multiple variance ratio test of Lo and MacKinlay [1988].

It is typical in such tests to allow for a linear trend. This is often necessary on economic grounds as well. For example, economic growth and inflation give rise to an upward long-run trend in stock prices. On the other hand, few of the tests mentioned above allow for changes or breaks to occur in the trend term. This is arguably a somewhat restrictive assumption, which may result

*This paper was substantially improved by the comments of two anonymous referees. We also thank Ba Chu, Robert de Jong, Lynda Khalaf, Thanasis Stengos, Shinichi Sakata, Yiguo Sun and participants at the 2009 Midwest Econometrics Group, the 3rd International conference on Computational and Financial Econometrics, the 2009 Canadian Econometric Study Group and the 2009 Canadian Economics Association Meetings for helpful comments and discussion. We take full responsibility for any remaining errors. This work was made possible by the facilities of the Shared Hierarchical Academic Research Computing Network (SHARCNET: www.sharcnet.ca).

${ }^{\dagger}$ Corresponding author: http://www.uoguelph.ca/ valexeev/ 
in unreliable inference. For example, changes to the trend growth rates or long-term average inflation rates would imply a break in trend for nominal stock prices.

An alternative perspective on the random walk test is to view it as a special case of a unit root test with uncorrelated errors. In the unit root testing literature there has been a long and ongoing interest in robustifying inference to the presence of structural breaks. Perron [1989] and Perron and Vogelsang [1992] demonstrate that structural breaks can cause difficulty for unit root tests, by causing an $\mathrm{I}(0)$ series with a break to resemble an $\mathrm{I}(1)$ process near the break point. Perron [1989] proposed the first unit root test that allows for the possibility of a break. His model allows for a break under both the null and alternative hypothesis, but with exogenously determined break dates. The next generation of tests, for example Zivot and Andrews [1992], Banerjee et al. [1992], allows for endogenously determined break dates under the alternative (but not the null) hypothesis. Most recently, Kim and Perron [2009] extend the earlier test of Perron [1994] to allow for a break in a trend function at an unknown time under both the null and alternative hypothesis.

A second issue that complicates unit root testing in the presence of structural breaks is the risk of misspecification with respect to the number of structural breaks. Vogelsang [1994] shows that the power of a unit root test is non-monotonic when a one-break model is estimated on data that contain two breaks. Lumsdaine and Papell [1997] consider testing the unit-root hypothesis, while allowing for two endogenous breaks under the alternative hypothesis. Ohara [1999] develops a unit root test that allows for an alternative hypothesis with multiple trend breaks of unknown dates. Kapetanios [2005] provides tests for the unit-root hypothesis against the occurrence of an unknown, but finite number of breaks.

In this paper we propose a level-crossing random walk test that is robust to the possible presence of multiple structural breaks. This approach builds on a rich literature involving level crossings and their application to random walk and unit root testing. Studies on level-crossings by continuous stationary Gaussian processes used in models of physical phenomena date back at least sixty years. Discretization of the continuous schemes and its applications to economics and finance begins with the works of Ho and Sun [1987], Burridge and Guerre [1996], and Ho and Hsing [1997] to name a few.

Interestingly, in perhaps the earliest examinations of the random walk (without drift) hypothesis, Cowles and Jones [1937] compared the frequency of sequences and reversals in historical stock returns. This could be interpreted as an informal level crossing test using a crossing level of zero.

Burridge and Guerre [1996] proposed a formal non-parametric unit root test based on the standardized number of level crossings of a random walk without deterministic terms (drift or trend). They observed that in the presence of a unit root, a time series, $x_{t}$, would only infrequently cross any crossing level $\mu$. They therefore used the empirical frequency of this event to distinguish between random walks and stationary processes. They showed that the asymptotic distribution of the test statistic relates to a scaled local time of a Brownian motion and found that the scale factor depends on the long-run variance of the process.

Garcia and Sanso [2006] extend the work of Burridge and Guerre [1996] in two aspects. First, they allow for more general structures of autocorrelated disturbances. Secondly, they allow for a linear time trend and propose a modified crossing statistic, based on the frequency with which $x_{t}$ crosses the time trend, which they estimate by $x_{1}+\left(\frac{x_{T}-x_{1}}{T}\right) t$, i.e. by fitting a line through the two sample endpoints. Thus, instead of using a fixed crossing level, their crossing level $\hat{\mu}_{t}$ follows the (estimated) linear trend, i.e. $\hat{\mu}_{t}=x_{1}+\left(\frac{x_{T}-x_{1}}{T}\right) t$. Equivalently, their procedure is based on the frequency with which the linearly detrended series, $x_{t}-x_{1}-\left(\frac{x_{T}-x_{1}}{T}\right) t$, crosses zero. 
Although Garcia and Sanso [2006] allow for a linear trend, neither they nor Burridge and Guerre [1996] allow for the possibility of trend breaks. While these methods work quite well in the no-break case for which they were designed, the crossing statistics constructed in these ways are susceptible to structural breaks. In Figure 1 the number of crossings, on which the Garcia and Sanso [2006] test statistic relies, will be equal to the number of crossings of the straight trend line and the stochastic process. It is apparent that for a stationary process with a single break in the middle, as depicted in the figure, the number of crossings will be minimal. The larger the magnitude of the break, the larger will be the loss in power and the greater will be size distortion. The tests are particularly sensitive to breaks occurring towards the middle of the sample.

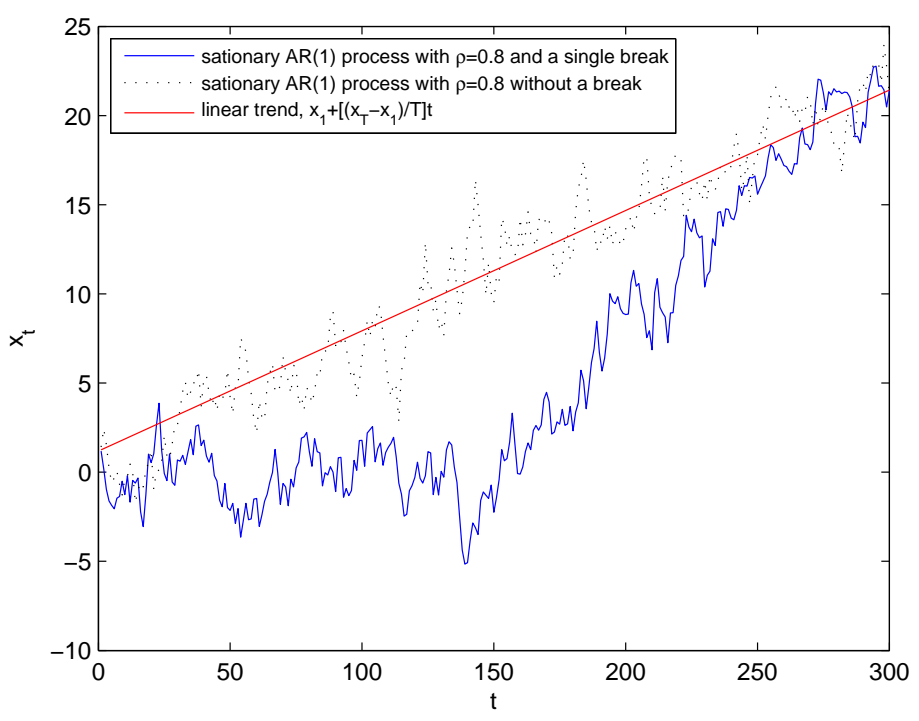

Figure 1: The dotted black line shows a simulated stationary series with a linear trend. The solid blue line shows a stationary series with a break in the slope of the trend in the middle of the data (at $t=150$, with a magnitude of 0.1). The straight solid red line shows an estimated trend line using the approach of Garcia and Sanso [2006].

We propose a level-crossing random walk test based on a modification of the Burridge and Guerre [1996] and Garcia and Sanso [2006] tests, in which their global crossing level, based on a constant or linear trend, is replaced by a more flexible local crossing level, based only on data in the temporal vicinity of $x_{t}$. More specifically, at each point in time $t$, we replace the linear trend of Garcia and Sanso [2006], $x_{1}+\left(\frac{x_{T}-x_{1}}{T}\right) t$, estimated using the two end points $x_{1}$ and $x_{T}$, by a local estimate of the trend given by $x_{t-m}+\left(\frac{x_{t+m}-x_{t-m}}{2 m}\right)(t-[t-m])=x_{t-m}+\frac{x_{t+m}-x_{t-m}}{2}$, where the slope, $\frac{x_{t+m}-x_{t-m}}{2 m}$, is based on the more temporally proximate 'local end points' $x_{t-m}$ and $x_{t+m}$. Analogously to Garcia and Sanso [2006] our test is based on the frequency with which $x_{t}$ crosses this localized crossing level. Equivalently, we define

$$
x_{t}^{(m)}=x_{t}-x_{t-m}-\frac{x_{t+m}-x_{t-m}}{2}, \quad \forall t=1 . . T, m=1 . .(T-1),
$$


as the locally detrended series, employing the out of sample boundary data points: ${ }^{1}$

$$
\begin{aligned}
x_{1-i} & =x_{1}+c(1-i), \\
x_{T+i} & =x_{T}+c(i), \quad \forall i=1 . . T-1,
\end{aligned}
$$

where $c=\frac{x_{T}-x_{1}}{T}$. Then we can re-express our test in terms of the frequency with which $x_{t}^{(m)}$ crosses zero. We refer to the parameter $m$ as a localization parameter, since it determines the degree to which the trend estimate is localized in time.

Figure 2 illustrates the ability of $x_{t}^{m}$ to purge much of the effect of the trend break, without a priori knowledge of the break location. It also shows the sensitivity of this detrending procedure to the choice of the localization parameter $m$. For the same simulated break-stationary series, $x_{t}$ used in Figure 1, it shows the linearly detrended series $x_{1}+\left(\frac{x_{T}-x_{1}}{T}\right) t$ employed by Garcia and Sanso [2006] alongside the locally detrended series $x_{t}^{m}$ for $m=5$ and 50. Due to the presence of the structural break, the linearly detrended series has an inverted U-shape and only crosses zero near the end point. Intuitively, because the detrending is global in nature, the structural break contaminates the entire detrending procedure, even though the break occurs at only one point in the series. The locally detrended series $x_{t}^{m}$ still show some contamination, displaying smaller inverted U-shapes. However, rather than affecting the entire series, this contamination is contained to the vicinity of the structural break. It can be seen from the figure that as the detrending becomes more heavily localized by decreasing $m$, the contamination from the structural break becomes both smaller and more tightly contained to the vicinity (in time dimension) of the structural break.

By localizing the crossing level in this way, the test is rendered robust to multiple trend breaks in either the intercept and/or the slope of the deterministic linear component. These breaks may occur under both the null and alternative hypothesis. Further, the test is nonparametric with respect to the breaks in the sense that we do not require knowledge of either the number or timing of the breaks. The test statistic is shown to have a standard normal null limit distribution, in both the presence and absence of trend breaks. Simulations also indicate that the test retains power in the presence of breaks.

An important practical consideration is the extent to which the crossing level is localized, a choice which is governed by the parameter $m$. Too strong a localization can negatively impact the power of the test, whereas too weak a localization may undermine the robustness of both size and power to the presence of trend-breaks. In the absence of a formal method for selecting $m$ based on asymptotic size and power criteria, we propose an informal resampling procedure that selects $m$ in order to maximize bootstrapped power in a proximate parametric model. Perhaps not surprisingly, small sample experiments indicated that this pre-selection can induce over-rejection, when employing the asymptotic critical values, which were derived under the assumption that $m$ is fixed. In an effort to account for the influence of selecting $m$, we propose critical values based on a second outer bootstrap in which the inner bootstrap (used to select the localization parameter) is repeated on each bootstrap replicate. Our Monte Carlo experiments suggest that this combined bootstrap approach results in both good size and power in practical settings. Establishing the theoretical properties of this multi-layered bootstrap approach is a non-trivial problem that lies outside the scope of this paper, but may provide an interesting topic for further research.

Our approach can be viewed as extending the level crossing approach of Burridge and Guerre [1996] and Garcia and Sanso [2006] to allow for breaks in trend. Where the number and timing of

\footnotetext{
${ }^{1}$ Out of sample boundary data points are required for $t<m+1$ or $t>T-m$ and are based on a linear extrapolation. See Section 2 for further discussion.
} 


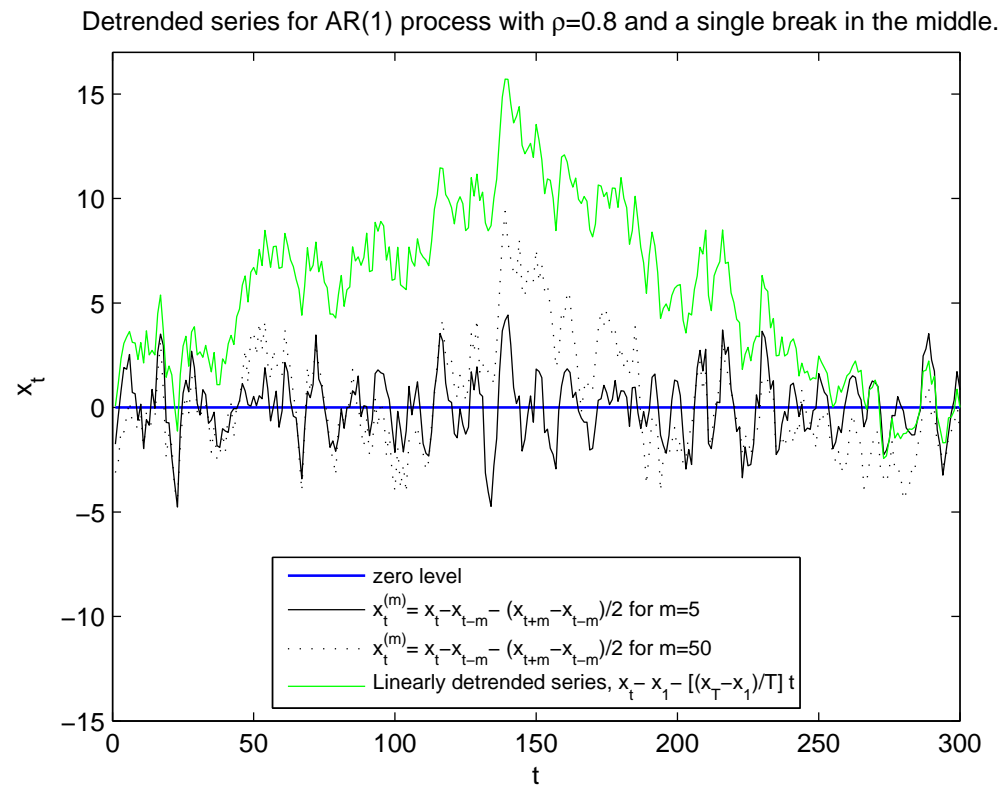

Figure 2: The solid straight horizontal line represents zero level. The solid black line shows the locally detrended series, $x_{t}^{(m)}$. It was obtained through equation (1) for $m=5$ using same series containing a break as in Figure 1. The dotted line, similarly, shows the locally detrended series, $x_{t}^{(m)}$, but for $m=50$. Finally, the top solid green line shows linearly detrended series, $x_{t}-x_{1}-\left(\frac{x_{T}-x_{1}}{T}\right) t$.

these beaks known a priori, one could envision a test based on the standardized crossings relative to a broken trend line. We instead take the view that such a broken trend is difficult to estimate with confidence and calculate crossings relative to a locally determined level. This allows the crossing level to respond quickly to breaks, leading to the robustness properties discussed above.

On the other hand, the robustness of the test with respect to structural breaks is not achieved without cost. The more localized is the crossing level, the higher the crossing frequency of the random walk and the less easily it is distinguished from a stationary process. For this reason, even in the presence of breaks, we find that the best test power is typically achieved when the crossing parameter is based on a moderately sized local region.

Localizing the crossing level also changes the nature of the large sample theory from nonstationary to stationary type asymptotics. Intuitively, deviations from localized trends are akin to gradual differences and thus after removing the trend component we effectively compare the crossings (about zero) of an appropriately differenced series to those of an over-differenced series. Since the crossing frequency of a stationary series depends on its serial correlation properties, our proposed test of random walk hypothesis, under which this correlation is restricted, has no natural unit root test counterpart. The crossing frequency is also sensitive to the distribution of the errors. Using the arcsine law, we derive the crossing frequencies for the family of elliptically symmetric distributions, which includes as special cases the normal, student-t, logistic, Laplace, and scaled mixed normal distributions. While this allows for the modeling of fat-tails, it is clear that the presence of asymmetries would alter the crossing rates. Finally, to the best of our knowledge, existing central limit theorems applicable to level crossings of stationary linear processes require i.i.d. innovations. However, in our simulations, we demonstrate the good performance of our test 
under both GARCH and EGARCH innovations, despite their violation of this assumption.

The plan of the paper is as follows: Section 2 briefly introduces the works previously done in the area of level crossings and proposes a random walk test based on the number of localized level crossings. Section 3 introduces the two bootstrap methods: an inner bootstrap to select the localization parameter and an outer bootstrap to select critical values. Section 4 studies finite sample size and power of the test and compares the proposed procedure to some existing representative unit root tests. Section 5 contains empirical applications to both Nelson-Plosser data set and Canadian interest and inflation rates. Finally, Section 6 concludes.

\section{Localized Level Crossing Random Walk Test}

Consider a sample $x_{1}, x_{2}, \ldots x_{T}$ from the process

$$
x_{t}=\mu_{t}+u_{t}, \quad u_{t}=\rho u_{t-1}+\varepsilon_{t}, \quad \text { for } t \geq 1
$$

and $u_{0}=0$, where $\mu_{t}$ is a deterministic component, discussed separately below. Equivalently, expressed as a nested equation, (3) can be represented as

$$
x_{t}=\rho x_{t-1}+\mu_{t}-\rho \mu_{t-1}+\varepsilon_{t} .
$$

The null and alternative hypothesis can then be stated as

$$
\begin{aligned}
& H_{0}: \quad \rho=1, \\
& H_{1}: \quad|\rho|<1 .
\end{aligned}
$$

In the absence of a trend (i.e. $\left.\mu_{t}=0\right)$ and with $\varepsilon_{t} \sim$ i.i.d. $\left(0, \sigma_{\varepsilon}^{2}\right)$, Burridge and Guerre [1996] define the normalized number of level crossings as:

$$
K_{T}(z)=T^{-1 / 2} \sum_{t=1}^{T}\left(\mathbf{1}\left[x_{t-1} \leq z, x_{t}>z\right]+\mathbf{1}\left[x_{t-1}>z, x_{t} \leq z\right]\right)
$$

where $z$ is a crossing level. They then define a level crossing random walk test statistic as:

$$
\varphi=\frac{\sqrt{T^{-1} \sum_{t=1}^{T}\left(\Delta x_{t}\right)^{2}}}{\widehat{M A D}} K_{T}(0)
$$

where $\widehat{M A D}=T^{-1} \sum_{t=1}^{T}\left|\Delta x_{t}\right|$ and $\Delta x_{t}=x_{t}-x_{t-1}$.

Garcia and Sanso [2006] generalize this procedure by allowing for the presence of linear trend, specified by $\mu_{t}=\alpha+\beta t$. They define a modified level crossing, say $K_{T}^{G S}$, by replacing $x_{t}$ in (5) with its detrended version $x_{t}-x_{1}-c t$, where $c=\frac{x_{T}-x_{1}}{T}$. In addition, they generalize the test-statistic in (6) to allow for residual serial correlation using

$$
\eta^{(G S)}=\frac{\widehat{\omega}_{\varepsilon, \varepsilon}}{\widehat{M A D}} K_{T}^{(G S)}(0)
$$

where $\widehat{\omega}_{\varepsilon, \varepsilon}$ is a consistent estimator of long-run variance of $\varepsilon_{t}$. They show that the null asymptotic distribution is standard Rayleigh and does not depend on the level $z$. 
As discussed in Section 1, neither the level crossing tests of Burridge and Guerre [1996] nor Garcia and Sanso [2006] are designed to accommodate structural breaks. In order to address this problem we define the normalized number of level crossings formula to allow for the presence of additive outlier-type structural breaks: sudden level shifts, changes in growth or both. We suppose that the deterministic process in (3) is given by a broken linear trend with $p$ breaks such that their corresponding break fractions, $\lambda_{i}$, are ordered increasingly: $0<\lambda_{1}<\lambda_{2}<\cdots<\lambda_{p}<1$. Thus, for any $i=1$..p,$\left\lfloor\lambda_{i} T\right\rfloor$ denotes the observation at which the $i$ th structural break occurs. Define the indicator for regime $i=1,2, \ldots, p$ by

$$
d_{i, t}= \begin{cases}0 & \text { for } t \leq \lambda_{i} T \\ 1 & \text { for } t>\lambda_{i} T\end{cases}
$$

Following Perron [1989], we generalize the deterministic process $\mu_{t}$ in (3) to allow for breaks in either the level and/or the slope of the linear trend:

$$
\begin{aligned}
\mu_{t}= & \alpha_{0}+\alpha_{1} d_{1, t}+\cdots+\alpha_{p} d_{p, t}+ \\
& +\beta_{0} t+\beta_{1}\left(t-\lambda_{1} T\right) d_{1, t}+\cdots+\beta_{p}\left(t-\lambda_{p} T\right) d_{p, t} .
\end{aligned}
$$

It is also useful to reformulate (9) in matrix notation. Let $\mathbf{A}=\left[\begin{array}{llll}\alpha_{0} & \alpha_{1} & \ldots & \alpha_{p}\end{array}\right]^{\prime}$ and $\mathbf{B}=$ $\left[\begin{array}{llll}\beta_{0} & \beta_{1} & \ldots & \beta_{p}\end{array}\right]^{\prime}$ be $(p+1) \times 1$ vectors of level and trend parameters respectively; and $\lambda=$ $\left[\begin{array}{llll}0 & \lambda_{1} & \ldots & \lambda_{p}\end{array}\right]^{\prime}$ be a $(p+1) \times 1$ vector of break fractions, with a first element of zero. Define $\mathbf{D U}_{\mathbf{t}}$ and $\mathbf{D} \mathbf{T}_{\mathbf{t}}$ as $1 \times(p+1)$ vectors as follows:

$$
\begin{aligned}
\mathbf{D U}_{\mathbf{t}} & =\left[\begin{array}{lllll}
1 & d_{1, t} & d_{2, t} & \ldots & d_{p, t}
\end{array}\right] \\
\mathbf{D T}_{\mathbf{t}} & =\left[\begin{array}{lllll}
t & \left(t-\lambda_{1} T\right) d_{1, t} & \left(t-\lambda_{2} T\right) d_{2, t} & \ldots & \left(t-\lambda_{p} T\right) d_{p, t}
\end{array}\right] .
\end{aligned}
$$

Then, in its matrix notation, $\mu_{t}$ in (9) can be represented as follows:

$$
\mu_{t}=\mathbf{D} \mathbf{U}_{\mathbf{t}} \cdot \mathbf{A}+\mathbf{D T}_{\mathbf{t}} \cdot \mathbf{B}
$$

This representation allows for multiple breaks in both level and trend. ${ }^{2}$

Since we assume that the timing, magnitude and number of breaks are unknown, we do not detrend based on (9). Instead, we employ the locally detrended series $x_{t}^{(m)}$ defined in (1), along with the out of sample boundary data points in (2) where $c=\frac{x_{T}-x_{1}}{T}$ as before. The out of sample boundary points are needed in order to calculate $x_{t}^{(m)}$ based on (1) when $t \leq m$ or $t>T-m$. They are constructed based on a simple extrapolation of the series on the left and right hand side taking into account the trend of the series. One reason for defining the out of sample points this way is that, as shown in Proposition 1, for $m=T-1$ the set up of the test is identical to Garcia and Sanso [2006]. Note that $\left(x_{T}-x_{1}\right) / T$ is a consistent estimator of $\beta$, when $\mu_{t}=\alpha+\beta t$ is a linear trend without break. ${ }^{3}$

Recall that in Garcia and Sanso [2006]'s model, discussed earlier, the process in (3) is detrended globally as follows: $x_{t}-x_{1}-c t$, where $c=\frac{x_{T}-x_{1}}{T}$. As shown in Proposition 1 below, this corresponds to the case in which $m=T-1$ in (1). We generalize this approach to allow for localized

\footnotetext{
${ }^{2}$ Note that case with no structural breaks is equivalent to $\mu_{t}=\mathbf{D U}_{\mathbf{t}} \cdot \mathbf{A}+\mathbf{D T}_{\mathbf{t}} \cdot \mathbf{B}=\alpha_{0}+\beta_{0} t$, and case with one structural break $\mu_{t}=\mathbf{D U}_{\mathbf{t}} \cdot \mathbf{A}+\mathbf{D} \mathbf{T}_{\mathbf{t}} \cdot \mathbf{B}=\alpha_{0}+\alpha_{1} d_{1, t}+\beta_{0} t+\beta_{1}\left(t-\lambda_{1} T\right) d_{1, t}$.

${ }^{3}$ Note also that this estimator is $T$-consistent for $\beta$ when $|\rho|<1$, but only $\sqrt{T}$-consistent when $\rho=1$. This follows because $\left(x_{T}-x_{1}\right) / T=\left(\mu_{T}-\mu_{1}\right) / T+\left(u_{T}-u_{1}\right) / T=\beta+O_{p}\left(T^{-1}\right)$ when $|\rho|<1$ and $\beta+O_{p}\left(T^{-1 / 2}\right)$ when $\rho=1$. The reduced convergence rate when $\rho=1$ is due to the fact that in this case $u_{T}=\sum_{t=1}^{T} \varepsilon_{t}=O_{p}\left(T^{1 / 2}\right)$.
} 
detrending. By introducing a localization parameter, $m$, we control the extent of the detrending of the process in (3). In Figure 2, the number of zero crossings of a locally detrended stochastic process with a single break, is represented by (11) discussed below.

A localized crossing is then said to occur if the locally detrended series $x_{t}^{(m)}$ crosses zero. The normalized number of local level crossings can then be defined as:

$$
\begin{aligned}
K_{T}^{(m)}(0)= & T^{-1 / 2} \sum_{t=1}^{T-1}\left(\mathbf{1}\left[x_{t}^{(m)} \leq 0, x_{t+1}^{(m)}>0\right]+\mathbf{1}\left[x_{t}^{(m)}>0, x_{t+1}^{(m)} \leq 0\right]\right), \\
& m=1 . .(T-1) .
\end{aligned}
$$

Equivalently, it can be expressed in following way:

$$
K_{T}^{(m)}(0)=T^{-1 / 2} \sum_{t=1}^{T-1} \mathbf{1}\left[x_{t}^{(m)} x_{t+1}^{(m)}<0\right], m=1 . .(T-1) .
$$

We next formalize the relation between this localized level crossing statistic and the crossing statistic of Garcia and Sanso [2006], which holds as a special case of (11), in which $m=T-1$.

Proposition 1 For $m=T-1$ the process in (1) becomes $x_{t}^{(T-1)}=x_{t}-x_{1}-c t$, with $c=\frac{x_{T}-x_{1}}{T}$. Then (11) is identical to the normalized number of level crossings, $K_{T}^{(G S)}(0)$, defined in Garcia and Sanso [2006].

Proof See appendix.

\subsection{Asymptotic properties of the centered partial sum in the absence of the deterministic component}

We first establish the asymptotic properties for the localized level crossing statistic when applied to random component $u_{t}$ in this subsection. In the following subsection, we then show that these same properties hold unchanged in the presence of the deterministic component, provided that the detrending is sufficiently localized. Similar to equations (1) and (11), we define

$$
u_{t}^{(m)}=u_{t}-u_{t-m}-\frac{u_{t+m}-u_{t-m}}{2}, \forall t=1 . . T,
$$

where $m$ is a fixed integer less than $T$ and

$$
K_{u, T}^{(m)}(0)=T^{-1 / 2} \sum_{t=1}^{T-1} \mathbf{1}\left[u_{t}^{(m)} u_{t+1}^{(m)}<0\right], m=1 . .(T-1) .
$$

Assumptions Many of the results below rely on one or more of the following assumptions:

$\mathscr{A} .1 \varepsilon_{t}$ is an i.i.d. process satisfying $\mathbb{E}\left[\varepsilon_{t}\right]=0$ and $\mathbb{E}\left[\varepsilon_{t}^{2}\right]=\sigma_{\varepsilon}^{2}<\infty$.

$\mathscr{A} .2 \varepsilon_{t}$ is a white noise process satisfying

$$
\mathbb{E}\left[\varepsilon_{t}\right]=0, \quad \mathbb{E}\left[\varepsilon_{t}^{2}\right]=\sigma_{\varepsilon}^{2}<\infty, \quad \mathbb{E}\left[\varepsilon_{t} \varepsilon_{s}\right]=0, \forall t \neq s .
$$

$\mathscr{A} .3 \varepsilon_{t}$ is a strictly stationary ellipsoidal process, i.e. its finite dimensional distributions have an elliptically symmetric density of the form $f(\varepsilon)=|\Sigma|^{-\frac{1}{2}} \phi\left(\varepsilon^{T} \Sigma^{-1} \varepsilon\right)$, where $\Sigma$ is the characteristic matrix and is proportional to $\operatorname{Cov}(\varepsilon)$. The functional form of $\phi($.$) is assumed$ to be unknown. 
$\mathscr{A} .4 \mathrm{~m}$ is a fixed positive integer.

$\mathscr{A} .5 p$ is a fixed positive integer.

$\mathscr{A} .6$ The characteristic function of $\varepsilon_{t}, \phi(t)=E[\exp (t \varepsilon \sqrt{-1})]$ satisfies $\int_{\infty}^{\infty}|\phi(t)|^{r} d t<\infty$ for some $r \in \mathbf{N}$.

The i.i.d. assumption on the innovations in $(\mathscr{A} .1)$ is required in existing central limit theorem results on level crossings for linear processes (see for example Ho and Hsing [1997], Hsing [1999],

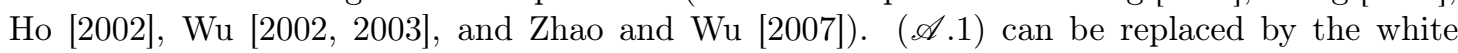
noise assumption $(\mathscr{A} .2)$ for most of our other results. The assumption in ( $\mathscr{A} .3)$ of elliptically symmetric innovations is required in order to employ the arcsine law to calculate the expected

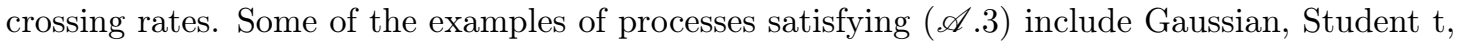
logistic, Laplace, and scaled mixed normal distributions, such as exponential power family distributions (see Fang et al. [1990], Tanaka and Shimizu [2001] for further discussion on symmetric type distributions). Therefore, assumption (A.3) allows for parametric distributions widely used to model fat tails. On the other hand $(\mathscr{A} .3)$ does not allow for asymmetries. The assumption of elliptical symmetry has been employed previously in areas of econometric theory (Hodgson [2006], Brown and Hodgson [2007]), asset pricing (Hodgson et al. [2002], Hodgson and Vorkink [2003]) and portfolio choice theory (see for example Owen and Rabinovitch [1983]). Assumption $(\mathscr{A} .4)$ ensures that the detrending is properly localized by preventing $m$ from growing with the sample size. The large sample behavior can change substantially when this assumption is violated - for the special case of $m=T-1$ see Garcia and Sanso [2006]. Assumption ( $\mathscr{A}$.5) requires the number of breaks to be finite. Assumption (AS.6) is required for asymptotic normality in Wu [2002]'s central limit theorem for functionals of linear processes.

We next derive the autocorrelation function for the locally detrended series $u_{t}^{(m)}$ in $(12)$.

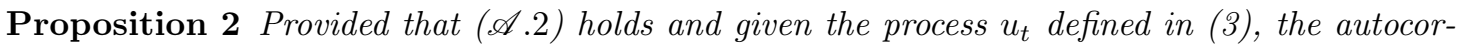
relation function, $r_{u, h}^{(m)} \equiv \gamma_{h}^{(m)} / \gamma_{0}^{(m)}=\mathbb{E}\left[u_{t}^{(m)} u_{t+h}^{(m)}\right] / \mathbb{E}\left[u_{t}^{(m)} u_{t}^{(m)}\right]$, for the process in (12) when $m<t<T-m$ is given by

for $|\rho|<1$ :

$$
r_{u, h}^{(m)}= \begin{cases}1 & h=0 \\ \frac{\rho^{h}}{2}+\frac{3 \rho^{h}+\rho^{2 m-h}-4 \rho^{m-h}}{2\left(1-\rho^{m}\right)\left(3-\rho^{m}\right)} & h=1 . . m \\ \frac{\rho^{h}}{2}+\frac{3 \rho^{h}-4 \rho^{h-m}+\rho^{2 m-h}}{2\left(\rho^{2 m}-2 \rho^{m}+1\right)+2 \rho^{3 h-3 m}-\rho^{3 h-2 m}} & h=m+1 . .2 m-1 \\ \frac{\rho^{h}}{2}+\frac{3 \rho^{h}-4 \rho^{m-h}+\rho^{h-m}}{2\left(1-\rho^{m}\right)\left(3-\rho^{m}\right)} & h \geq 2 m\end{cases}
$$

for $\rho=1$ :

$$
r_{u, h}^{(m)}= \begin{cases}1 & h=0 \\ \frac{2 m-3 h}{2 m} & h=1 . . m \\ \frac{m-h}{2 m} & h=m+1 . .2 m-1 \\ 0 & h \geq 2 m\end{cases}
$$

Proof See appendix.

We next solve for the population crossing rate in terms of the first order autocorrelation. 


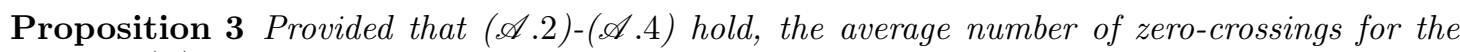
process $u_{t}^{(m)}$ as defined by (12) is:

$$
\mathbb{E}\left(\mathbf{1}\left[u_{t}^{(m)} u_{t+1}^{(m)}<0\right]\right)=\frac{1}{\pi} \cos ^{-1} r_{u, 1}^{(m)} .
$$

Proof See appendix.

This result is an application of the arcsine formula for zero mean symmetric elliptical processes given by Barnett [1996] and Tanaka and Shimizu [2001], which is a generalization of Rice's formula for Gaussian processes. The crossing rate in (15) does not depend on $\sigma_{\varepsilon}$, since we only consider crossings about zero, the mean of $u_{t}^{m}$, which is also the median due to the symmetry assumption in $(\mathscr{A} .3)$. When constructing the test statistic later in this section, we will require an explicit expression for the population crossing rate under the null hypothesis of a random walk $(\rho=1)$.

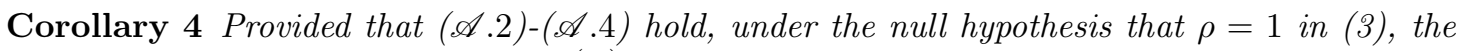
autocorrelation function is given by $r_{u, 1}^{(m)}=(2 m-3) /(2 m)$ and the average crossing rate for the process $u_{t}^{(m)}$ equals $\frac{1}{\pi} \cos ^{-1} \frac{(2 m-3)}{2 m}$.

The result of Corollary 4 is an immediate application of Propositions 2 and 3.

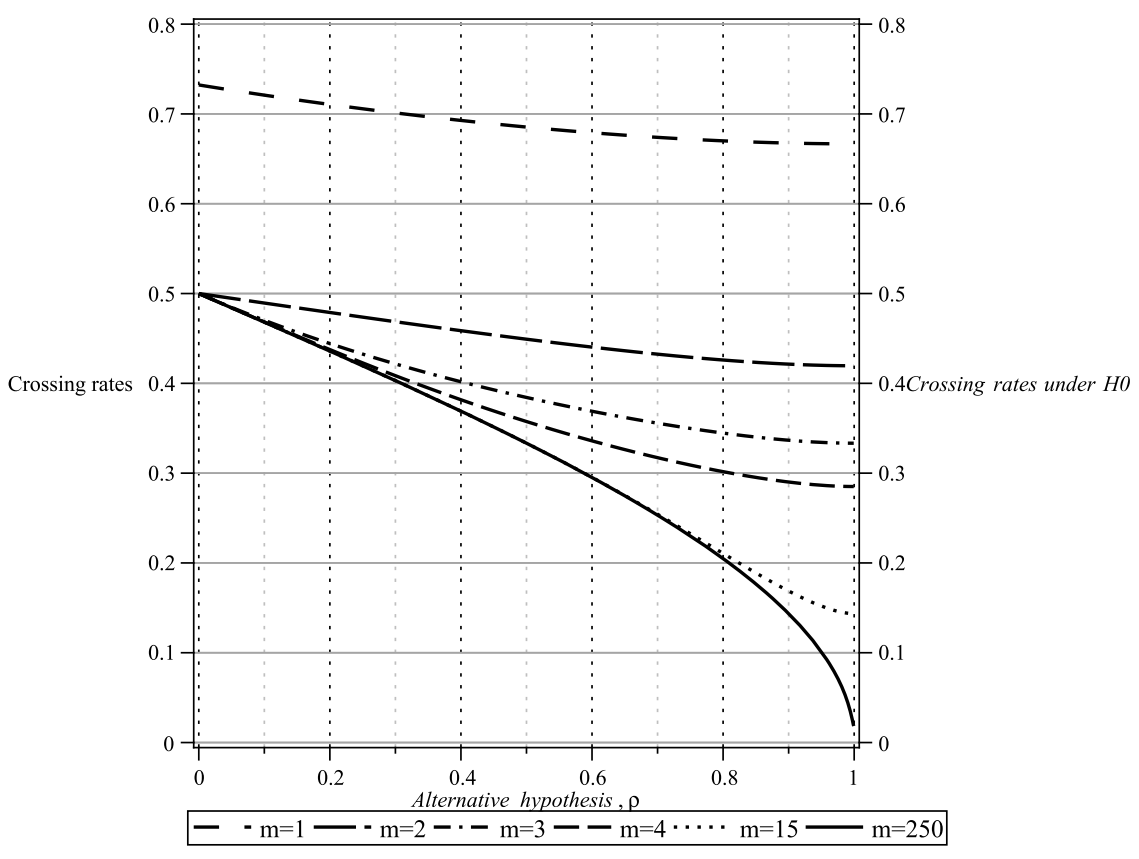

Figure 3: Level crossing rates, $\mathbb{E}\left(\mathbf{1}\left[u_{t}^{(m)} u_{t+1}^{(m)}<0\right]\right)$, under both the null and alternative hypothesis for selected values of the localization parameter $m$. The value of the autoregressive parameter $\rho \in[0,1]$ is displayed on the horizontal axis. The vertical axis provides the corresponding crossing rates.

Figure 3 depicts average crossing rates as functions of $\rho$ for several values of $m$ : from a minimum $m=1$ to a maximum $m=250$ with several cases in between. It is apparent, that higher 
values of $m$ result in a greater distinction between the null $(\rho=1)$ and alternative $(0 \leq \rho<1)$ hypotheses. To make the point more transparent, consider Figure 4, where we have plotted the average crossing rate as a function of $m$ for $\rho=1$ and $\rho=0.85$.

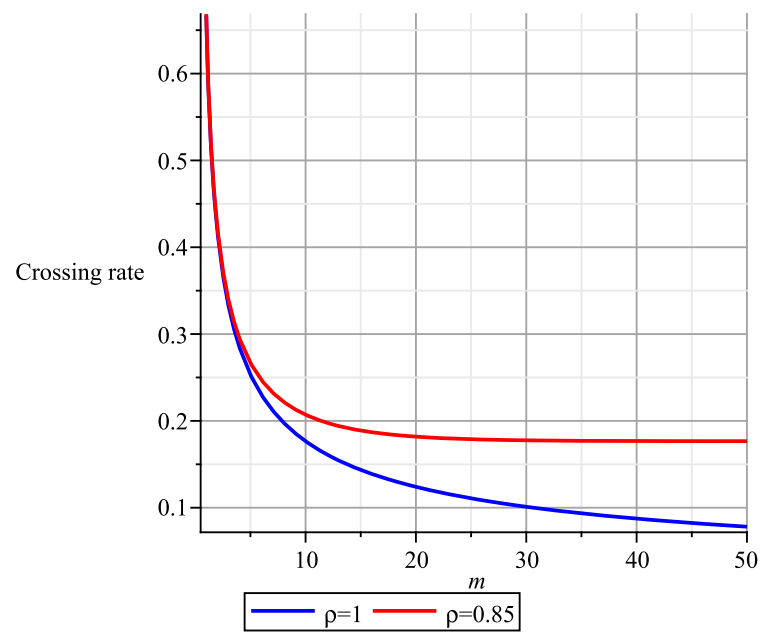

Figure 4: Level crossing rates as a function of the localization parameter $m, \mathbb{E}\left(\mathbf{1}\left[u_{t}^{(m)} u_{t+1}^{(m)}<0\right]\right)$, under both the null hypotheses of random walk $\rho=1$ and a single point alternative $\rho=0.85$.

The following result establishes asymptotic normality for the normalized number of crossings.

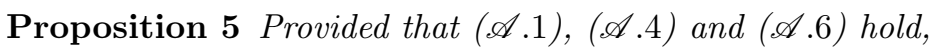

$$
K_{u, T}^{(m)}(0)-\mathbb{E}\left[K_{u, T}^{(m)}(0)\right] \stackrel{d}{\rightarrow} N\left(0, \omega_{\mathbf{1}_{u}, \mathbf{1}_{u}}^{(m)}\right)
$$

for some asymptotic variance $\omega_{\mathbf{1}_{u}, \mathbf{1}_{u}}^{(m)}<\infty$.

Proof See appendix.

This result follows as an application of $\mathrm{Wu}[2002]$ who provides a central limit theorem for functionals of linear processes with i.i.d. innovations, extending the earlier results of Ho and Sun [1987] for non-instantaneous filters of a stationary Gaussian processes.

Under the restriction of the null hypothesis $(\rho=1)$, we next show that $\omega_{\mathbf{1}_{u}, \mathbf{1}_{u}}$ can be expressed in terms of the long-run variance of the crossing indicator $\mathbf{1}\left[u_{t}^{(m)} u_{t+1}^{(m)}<0\right]$.

Proposition 6 Provided that $(\mathscr{A} .1),(\mathscr{A} .4)$ and $(\mathscr{A} .6)$ hold and under the null hypothesis that $\rho=1$, the asymptotic variance in Proposition 5 is given by

$$
\begin{aligned}
\omega_{\mathbf{1}_{u}, \mathbf{1}_{u}}^{(m)} & =\sum_{h=-\infty}^{\infty} \gamma_{\mathbf{1}[\cdot]}(h) \\
& \text { where } \gamma_{\mathbf{1}[\cdot]}(h) \equiv \operatorname{Cov}\left[\mathbf{1}\left[u_{t}^{(m)} u_{t+1}^{(m)}<0\right], \mathbf{1}\left[u_{t+h}^{(m)} u_{t+1+h}^{(m)}<0\right]\right] .
\end{aligned}
$$

Proof See appendix.

Remark Under the null hypothesis, when $m=1$ and $(\mathscr{A} .2)$ and $(\mathscr{A} .3)$ hold, $\omega_{\mathbf{1}_{u}, \mathbf{1}_{u}}^{(m)}=\frac{8}{45}$, can be calculated using binomial probabilities. ${ }^{4}$ However, as discussed in Sinn and Keller [2010],

\footnotetext{
${ }^{4}$ Details of the calculations are available upon request.
} 
explicit calculations are no longer available for $m>1$, since it involves estimation of $\gamma_{\mathbf{1}[\cdot]}(h)$ for any $h>1$. In the Gaussian case, it follows from Ho and Hsing [1997] that

$$
\omega_{\mathbf{1}_{u}, \mathbf{1}_{u}}^{(m)}=\sum_{j=q}^{\infty} \frac{h_{j}^{2}}{j !} \sum_{s=-\infty}^{\infty}\left(r_{u, s}^{(m)}\right)^{j}
$$

where $h_{j}=\int 1[x<0] H_{j}(x) d \Phi(x)$ is a truncated Hermite expansion of the indicator function with power $\operatorname{rank}^{5} q$ and $H_{j}(x)=(-1)^{j} e^{x^{2} / 2}\left(d^{j} e^{-x^{2} / 2} / d x^{j}\right)$. However, the use of this formula still entails numerical approximation. An alternative numerical approximation is provided by Sinn and Keller [2010]. In light of these complications, we instead estimate $\omega_{\mathbf{1}_{u}, \mathbf{1}_{u}}^{(m)}$ using a standard heteroskedasticity autocorrelation consistent (HAC) variance estimator of the form

$$
\begin{aligned}
\hat{\omega}_{\mathbf{1}_{u}, \mathbf{1}_{u}} & =\sum_{j=-T+1}^{T-1} k\left(j / S_{T}\right) \hat{\gamma}_{\mathbf{1}}^{(m)}(j), \text { where } \\
\hat{\gamma}_{\mathbf{1}}^{(m)}(j) & =T^{-1} \sum_{t=|j|+1}^{T-1} \hat{v}_{t+1}^{(m)} \hat{v}_{t+1-|j|}^{(m)}, \\
\hat{v}_{t+1}^{(m)} & =\mathbf{1}\left[u_{t}^{(m)} u_{t+1}^{(m)}<0\right]-T^{-1} \sum_{t=1}^{T-1} \mathbf{1}\left[u_{t}^{(m)} u_{t+1}^{(m)}<0\right]
\end{aligned}
$$

$k$ is a kernel weight function, and $S_{T}$ is a bandwidth parameter (see e.g. Newey and West [1987], Andrews [1991]),

With these results in hand, we provide a $t$-type test statistic with a standard normal null asymptotic distribution.

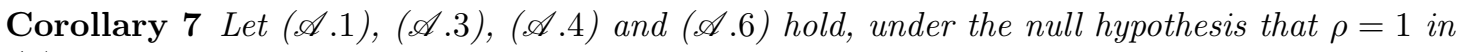
(3). Then:

(i) For any consistent estimator $\hat{\omega}_{\mathbf{1}_{u}, \mathbf{1}_{u}}$ of $\omega_{\mathbf{1}_{u}, \mathbf{1}_{u}}>0$

$$
t_{u}^{(m)}=\frac{K_{u, T}^{(m)}(0)-\sqrt{T} \cdot \frac{1}{\pi} \cos ^{-1} \frac{(2 m-3)}{m}}{\sqrt{\hat{\omega}_{\mathbf{1}_{u}, \mathbf{1}_{u}}^{(m)}}} \stackrel{d}{\rightarrow} N(0,1) .
$$

(ii)If additionally $\hat{\omega}_{\mathbf{1}_{u}, \mathbf{1}_{u}}$ is defined by (17) where $k()$ satisfies $\int_{-\infty}^{\infty}|k(x)| d x<\infty$ and belongs to the class of kernels $\mathcal{K}_{1}$ defined in Andrews [1991], $S_{T} \rightarrow \infty$ and $S_{T} / T \rightarrow 0$ then $\hat{\omega}_{\mathbf{1}_{u}, \mathbf{1}_{u}}$ is a consistent estimator for $\omega_{\mathbf{1}_{u}, \mathbf{1}_{u}}^{(m)}$.

Proof See appendix.

What follows from the above corollary is that in the absence of a deterministic component, one could employ (18) to test the random walk null hypothesis (without deterministic component). One should note, however, that this approximation, based on the assumption ( $\mathscr{A} .4)$ that $m$ is fixed, deteriorates as $m$ increases relative to $T$ in finite samples. For example, when $m=T-1$ Assumption $(\mathscr{A} .4)$ is not met. In this case, using Proposition 1 and the normalized number of zero crossings defined in (11) one can directly apply the result of Garcia and Sanso [2006] to show that the underlying test statistic will follow the standard Rayleigh distribution.

\footnotetext{
${ }^{5}$ Function $K$ has power rank $q$ for some positive integer $q$ if $K_{\infty}^{(q)}(0)$ exists and is nonzero and $K_{\infty}^{(r)}(0)=0$ for $1 \leq r<q$, where $K_{\infty}^{(r)}(x)=\frac{d^{r}}{d x^{r}} \int K(x+y) d F(y)$ whenever it is defined (see e.g. Ho and Hsing [1997], p. 1640 and Hsing [2000], p. 24). In the case of Gaussian process, $q$ is simply the Hermite rank. We say that the Hermite rank of $K$ is the index of the first non-zero coefficient in the expansion of $K$ in Hermite polynomials (see e.g. Taqqu [1979], Csörgó and Mielniczuk [1996]).
} 


\subsection{Asymptotic invariance to the presence of the deterministic com- ponent}

The results in the subsection above were defined for the random component $u_{t}$. In this section, we first show that, when the deterministic component $\mu_{t}$ is a linear trend, without breaks, $x_{t}^{(m)}=u_{t}^{(m)}$. Therefore the statistics discussed above are numerically unchanged with the addition of a linear trend. Intuitively, $x_{t}^{(m)}$ eliminates the trend, via a gradual differencing.

We next show that when $\mu_{t}$ is a linear trend with a finite number of breaks $x_{t}^{(m)}$ and $u_{t}^{(m)}$ differ at only $\max (2 m+1) p$ number of points. Because the level crossing statistics involve bounded indicator functions, this implies a tight bound on the discrepancy between the statistics based on the processes with and without the structural breaks.

Substituting for $x_{t}, x_{t-m}$ and $x_{t+m}$ from (3) into (1) yields:

$$
\begin{aligned}
x_{t}^{(m)}= & {\left[\mu_{t}+u_{t}\right]-\left[\mu_{t-m}+u_{t-m}\right] } \\
& -\frac{\left[\mu_{t+m}+u_{t+m}\right]-\left[\mu_{t-m}+u_{t-m}\right]}{2} .
\end{aligned}
$$

Equivalently,

$$
x_{t}^{(m)}=u_{t}-u_{t-m}-\frac{u_{t+m}-u_{t-m}}{2}+D C_{t}=u_{t}^{(m)}+D C_{t},
$$

where

$$
D C_{t}=\mu_{t}-\mu_{t-m}-\frac{\mu_{t+m}-\mu_{t-m}}{2} .
$$

The test statistic based on (11) will be invariant to the breaks and trend/level parameters as long as $D C_{t}=0$.

For the simple case with no structural changes in the level or linear trend, $\mu_{t}=\alpha_{0}+\beta_{0} t$, we can show that $D C_{t}=\beta_{0} m-\frac{2 \beta_{0} m}{2}=0$ for any values of $\alpha_{0}, \beta_{0}, m$ and any sample size $T$. And thus the test statistics based on (11) will be numerically equivalent to the test statistics based on (13), irrespective of the magnitude of the level and trend parameters.

This exact equivalence no longer holds in the presence of trend-breaks. However, the break only affects those local level crossings for which the break point is included in the local detrending. This bounds the rate of discrepancy between $\mathbf{1}\left[u_{t}^{(m)} u_{t+1}^{(m)}<0\right]$ and $\mathbf{1}\left[x_{t}^{(m)} x_{t+1}^{(m)}<0\right]$.

\section{Proposition 8}

$$
\left|K_{T}^{(m)}-K_{u, T}^{(m)}\right| \leq T^{-1 / 2}(2 m+1) p .
$$

Proof See appendix.

Under Assumptions ( $\mathscr{A} .4)$ and $(\mathscr{A} .5)$, both $m$ and $p$ are fixed and therefore $\lim _{T \rightarrow \infty}(2 m+1) p / \sqrt{T}=$ 0 and $K_{T}^{(m)}$ is asymptotically equivalent to $K_{u, T}^{(m)}$. If we allow $m$ or $p$ to grow with the sample size, $K_{T}^{(m)}$ and $K_{u, T}^{(m)}$ will be asymptotically equivalent provided that $m p=o_{p}\left(T^{1 / 2}\right)$. This implies robustness of the test to the presence of breaks.

An important case for which the equivalence of $K_{T}^{(m)}$ and $K_{u, T}^{(m)}$ is no longer implied by Proposition 8 is when the process is detrended globally by setting $m=T-1$ as in Garcia and Sanso 
[2006]. This is because $m$ grows too quickly as a function of $T$ for the bound in Proposition 8 to be meaningful.

\section{Bootstrap selection of $m$ and bootstrap critical values}

The last two sections derived a formal asymptotic test of the random walk hypothesis, robust to the presence of structural breaks, based on the localized level crossings, under the assumption that the degree of localization $m$ was fixed. However, the practical implementation of the test requires a choice of $m$. Moreover, if the selection of $m$ is itself subject to noise, this may change the distribution of the test statistic, whose asymptotic critical values are derived under the assumption that $m$ is fixed. In this section we propose an informal computational procedure designed to select $m$ in a way that should provide reasonably good power in most cases, while attempting to control the overall size of the test by adjusting the critical values to take into account the pre-selection of $m$. While this combined computational procedure has a clear rationale, which is borne out by its good performance in the simulations of Section 4, it entails a multi-layered bootstrap whose theoretical properties are not easily derived using the standard argument that apply to more standard bootstrap applications. One might therefore cautiously describe these as computationally sophisticated rules of thumb.

The choice of $m$ involves two countervailing influences on power: robustness to breaks and discrimination between the null and alternative hypotheses in the absence of breaks. Smaller values of $m$ will result in a random walk test robust to the presence of multiple structural breaks and their magnitudes. Figure 5 presents empirical rejection rates of the LLC test for a user specified parameter $m$. To illustrate the point, several values of $m$ were selected and several single break models were considered under the alternative hypothesis (with the magnitude of the break, $\beta$, on the horizontal axis). As can be seen from Figure 5, the LLC test with $m=1$ or $m=2$ is robust to the magnitude of the break, but suffers from low power. At the same time, very large values of $m$ (e.g $m=T-1$ ) result in higher rejection rates in the absence of a break. The power drops quickly as the magnitude of the break increases, giving rise to the inverted U-shapes seen in Figure 5.

Figures 6 and 7 show sensitivity of the test power to changes in break location, $\lambda$, for a range of the localization parameters, $m$, for sample sizes of $T=100$ (Figure 6) and $T=500$ (Figure 7). The test is robust to the location of the break for smaller values of $m$, but experiences significant power loss for larger values of $m$ when the break is located close to the middle of the series.

On the other hand, in the absence of breaks, a reduction in $m$ leads to a reduction in test power due to the smaller difference between the average number of crossing rates under the null and alternative hypotheses (see Figure 4). When breaks are present, our simulations indicate a nonmonotonic relationship between test power and the magnitude of $m$. In this case, the best power is generally obtained for intermediate values of $m$. This is observed in the Figures 5-7 discussed above. The straight lines towards the top of Figure 5 illustrate a range on intermediate values of $m$ for which the test shows good power regardless of the break magnitude $(\beta \mathrm{s})$. Likewise, the surfaces in Figures 6 and 7 show a range of intermediate values for $m$ that result in good power regardless of the break location.

We suggest a computational algorithm for selecting the parameter $m$, based on a finite sample power criterion. Since the finite sample power depends on the true but unknown model for the data generating process, we employ a proximate model in the spirit of Andrews [1991] to perform 


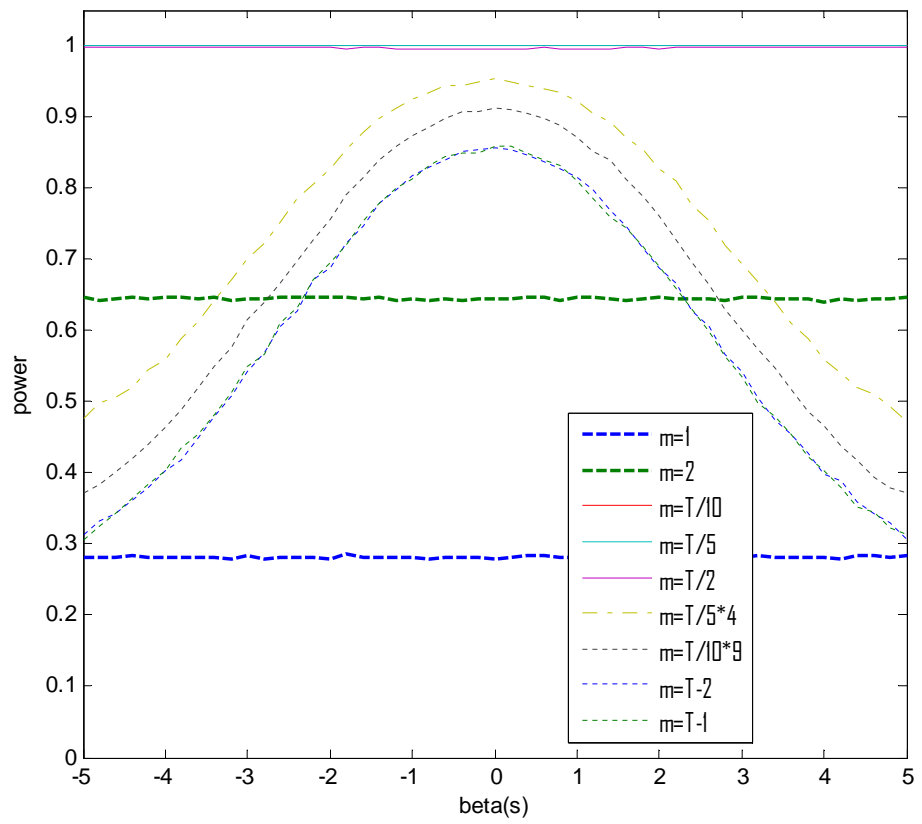

Figure 5: Sensitivity of the test power to the magnitude of the structural break for different choices of the localization parameter $m$. The vertical axis shows rejection rates under the alternative hypothesis for $\rho=0.5$, $T=500$, Gaussian errors and $\mathbf{A}=[0 ; 0], \mathbf{B}=\left[0 ; \beta_{1}\right]$ and $\lambda=[0 ; 0.5]$. The magnitude of the break, $\beta_{1}$, is varied along the horizontal axis.

this comparison. ${ }^{6}$ For our proximate model we employ an AR(1) with resampled estimated errors and a maximum of $\bar{p}$ breaks in both the shift and trend parameters:

$$
\begin{aligned}
x_{t} & =\hat{\rho} x_{t-1}+\hat{\mu}_{t}-\hat{\rho} \hat{\mu}_{t-1}+\varepsilon_{t} \\
\hat{\varepsilon}_{t} & \equiv x_{t}-\hat{\rho} x_{t-1}-\left(\hat{\mu}_{t}-\hat{\rho} \hat{\mu}_{t-1}\right) \\
\hat{\mu}_{t} & =\widehat{\mathbf{D U}}_{\mathbf{t}} \cdot \hat{\mathbf{A}}+\widehat{\mathbf{D T}}_{\mathbf{t}} \cdot \hat{\mathbf{B}}
\end{aligned}
$$

In order to estimate our proximate model, we first estimate the bottom equation in (20) using the procedure of Bai and Perron [1998] to estimate the number and location of breaks. We then estimate the autoregressive component of the proximate model conditional on the estimated number and location of breaks. Next, we repeatedly resample the residuals of the estimated proximate model in order to provide a bootstrap based approximation to the power of the test for each value of $m$. Finally, we select the value of $m$ that maximizes the power in our proximate model.

The estimation of the proximate model and the selection of $m$ adds additional estimation error not accounted for in the asymptotic distributions based on the assumption that $m$ is non-random. Selecting $m$ to maximize power may also lead to size distortion. To address these issues, we attempt to control the overall test size using bootstrapped critical values in which we re-estimate the proximate model and re-select $m$ for each bootstrap replication. We perform this bootstrap

\footnotetext{
${ }^{6}$ We refer to this as a 'proximate' model because we use it to provide an approximation rather than assuming it to be a true model. From a pragmatic perspective, one hopes that the proximate model will deliver a reasonable intermediate value of $m$ even if it is misspecified or suffers from estimation error.
} 


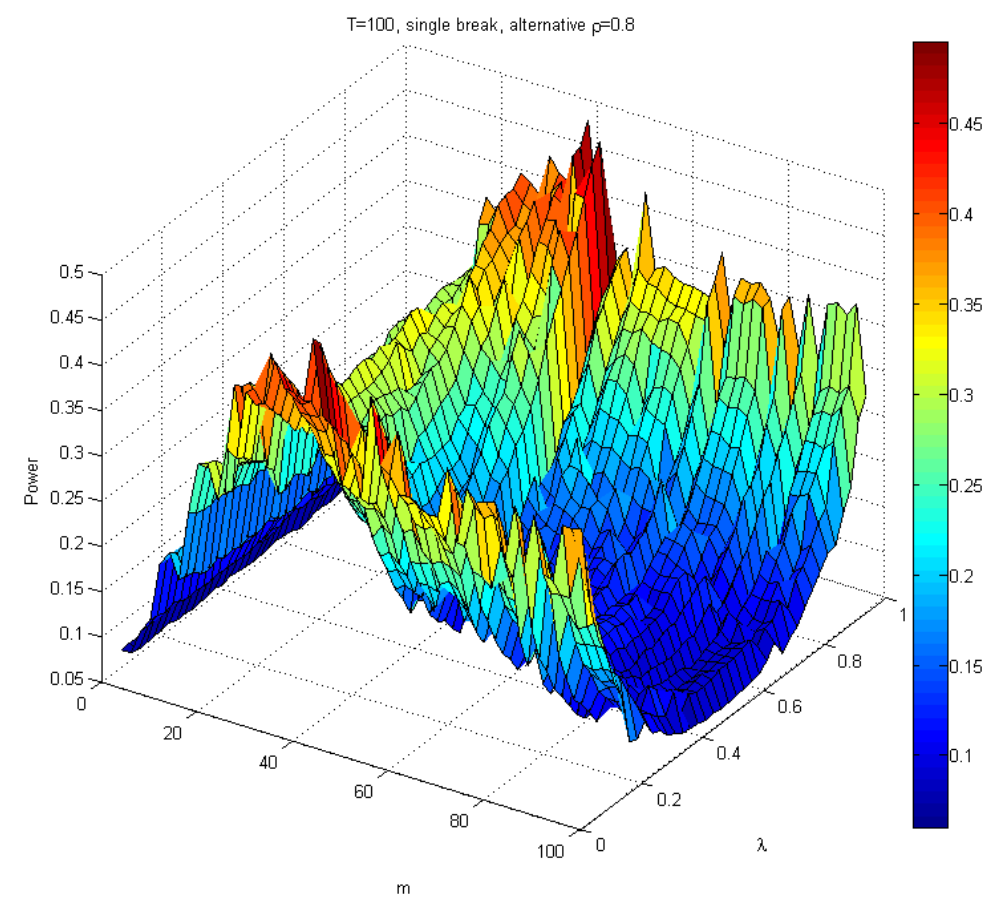

Figure 6: Sensitivity of the test power to changes in break location, $\lambda$, for different choices of the localization parameter $m$. Constructed under the alternative hypothesis based on a model with a single break in trend using $\rho=0.8, \mathbf{A}=[0 ; 0], \mathbf{B}=[0 ; 5], \lambda=\left[0 ; \lambda_{1}\right]$ and $T=100$, where we allow $\lambda_{1}$ to vary in $[0.05, .95]$. Computed using 5000 replications.

by resampling the residuals of the following restricted version of our proximate model

$$
\begin{aligned}
x_{t} & =x_{t-1}+\hat{\mu}_{t}-\hat{\mu}_{t-1}+\varepsilon_{t} \\
\tilde{\varepsilon}_{t} & \equiv x_{t}-x_{t-1}-\left(\hat{\mu}_{t}-\hat{\mu}_{t-1}\right) \\
\hat{\mu}_{t} & =\widehat{\mathbf{D U}}_{\mathbf{t}} \cdot \hat{\mathbf{A}}+\widehat{\mathbf{D T}}_{\mathbf{t}} \cdot \hat{\mathbf{B}},
\end{aligned}
$$

in which we enforce the null hypothesis by setting $\rho=1$ in equation (4). In this way we attempt to capture the effect of endogenously selecting $m$ in our bootstrap replications. To be specific, we use the following procedures to select $m$ and to select critical values in order to control overall test size:

\subsection{Procedure to select $m^{*}$}

1. Given the data, we estimate ${ }^{7}$ the parameters of the unrestricted proximate model described by equations (4) and (10) to obtain the fitted versions of both the unrestricted, (20), and restricted, (21), models.

2. Then, for every $m=1, \ldots, T-1$ we:

\footnotetext{
${ }^{7}$ We use Bai and Perron [1998] procedure to estimate the number and location of the breaks in the proximate model.
} 


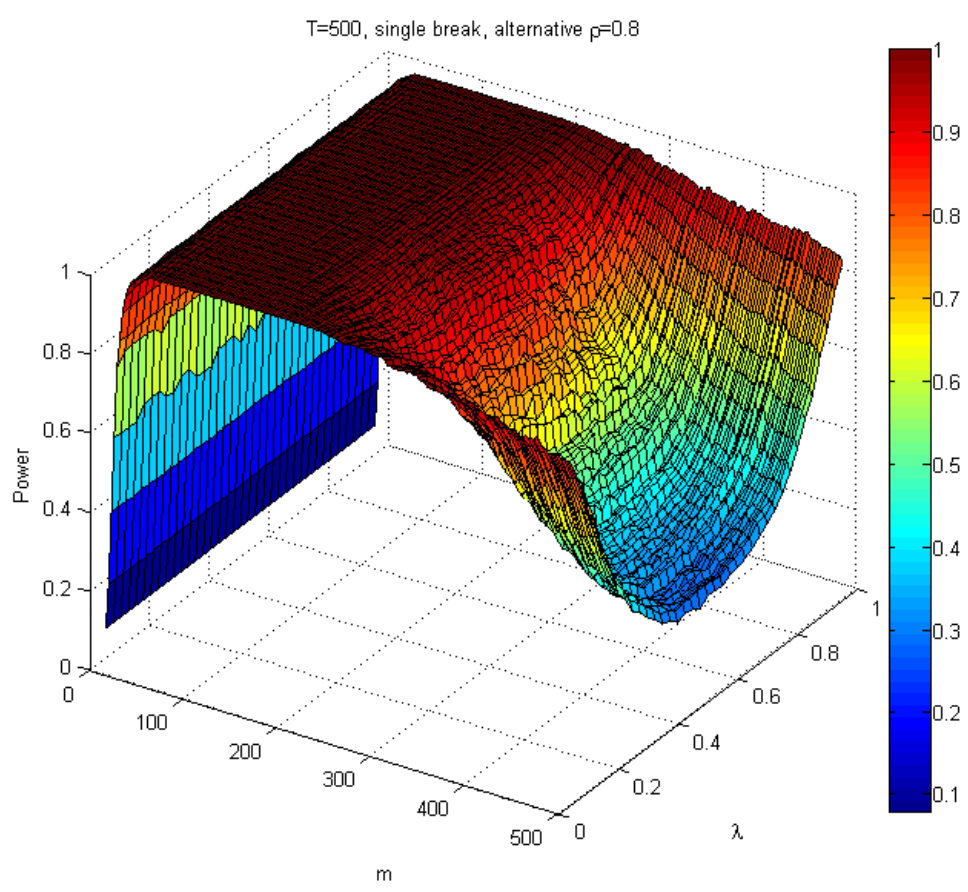

Figure 7: Sensitivity of the test power to changes in break location, $\lambda$, for different choices of the localization parameter $m$. Constructed under the alternative hypothesis based on a model with a single break in trend using $\rho=0.8, \mathbf{A}=[0 ; 0], \mathbf{B}=[0 ; 5], \lambda=\left[0 ; \lambda_{1}\right]$ and $T=500$, where we allow $\lambda_{1}$ to vary in $[0.05, .95]$. Computed using 5000 replications.

(a) Bootstrap $M$ series of length $T$ from the restricted model (21) where we set $\rho=1$ and the other parameters are estimated in Step (1), and where $\{\tilde{\varepsilon}\}$ are sampled with replacement from $\{\hat{\varepsilon}\}$.

(b) Calculate the normalized number of crossings, $K_{T}^{(m)}(0)$ for each of the $M$ series in Step (2a).

(c) Find a one tailed $(1-\alpha)$ critical value for $K_{T}^{(m)}(0)$ based on the $\alpha$ quantile of the bootstrap replicates $K_{T}^{(m)}(0)$.

(d) Bootstrap $M$ series from the unrestricted model using (20) estimated in Step (1), where $\left\{\tilde{\varepsilon}_{t}\right\}$ are sampled with replacement from $\left\{\hat{\varepsilon}_{t}\right\}$ of the same length.

(e) Form the test statistic for each bootstrap replicate and using the critical values in Step (2c), find the rejection rate.

3. Choose $m^{*}$ corresponding to the value of $m$ with largest rejection rate in Step (2e).

\subsection{Selection of critical values to control overall test size}

1. Given the data, we estimate parameters of the unrestricted proximate model described by equation (20).

2. Bootstrap $M$ series from the restricted model (21) where we impose the null hypothesis by setting $\rho=1$. The other parameters are estimated from the unrestricted model in Step (1) and $\left\{\tilde{\varepsilon}_{t}\right\}$ are sampled with replacement from $\left\{\hat{\varepsilon}_{t}\right\}$ of the same length. 
3. Select $m^{*}$ using the procedure in Section 3.1 for each of the $M$ series in (2). Then calculate the normalized number of crossings, $K_{T}^{\left(m^{*}\right)}(0)$ for each of the $M$ series.

4. Find $(1-\alpha)$ critical value (one tailed) using the $\alpha$ quantile of the $M K_{T}^{\left(m^{*}\right)}(0)$ replicates in Step (3).

Given the complicated, multi-layered nature of the bootstrap procedure discussed above, the formal justification of its validity is a nontrivial problem, which lies outside the scope of the current paper. However, the Monte Carlo experiments reported in Section 4 suggest that it has the potential to work well in practice.

\section{Finite Sample Size and Power}

In this section we conduct a small sample simulation study in order to assess the empirical size and power of our test. We use (4) and (10) as the data generating process in our simulations. The sample size is set to 250 . We consider three cases: (i) a case without a break where $\mathbf{A}=[0]$, $\mathbf{B}=[0], \lambda=[0]$; (ii) a case where the break in the trend slope is present in the middle of the series, for which $\mathbf{A}=[0 ; 0], \mathbf{B}=[0 ; 0.1], \lambda=[0 ; 0.5]$; (iii) and a case with two breaks in the trend slope, equally spaced at $1 / 3 T$ and $2 / 3 T$, specifically $\mathbf{A}=[0 ; 0 ; 0], \mathbf{B}=[0 ; 0.1 ; 0.2]$, $\lambda=[0 ; 0.33 ; 0.66]$.

The performance of the Localized Level Crossing test (LLC) proposed above is compared to the performance of the Garcia and Sanso [2006] testing procedure (GS) which is not adapted for structural breaks. We also compare to the Zivot and Andrews [1992] testing procedure $\left(\mathrm{ZA}^{8}\right)$, which allows for a single endogenous break under the alternative hypothesis. We include the results for Kim and Perron [2009] procedure (KP). The KP test allows for a single endogenous break under both the null and alternative hypotheses. Finally, we report the size and power for the Augmented Dickey-Fuller test as an additional benchmark. ${ }^{9}$ We compute our results using 5,000 simulations for the ADF, ZA, KP and GS tests. For the LLC test we employ only 1,000 simulations, since it is more computationally demanding. Within the LLC test procedure we use 1,000 replications for the inner bootstrap used to select $m^{*}$ and 1,000 simulations for the outer bootstrap procedure used for selecting critical values to control overall test size.

The results from our baseline simulations using Gaussian innovations are shown in Table 1. Our simulation experiments show that, in the absence of any structural breaks, the ADF test outperforms all of the other tests, as expected (columns 2-5). The LLC test outperformed the GS test in this scenario. We conjecture that this improvement is due to the fact that the LLC test selects $m$ to maximize power.

Both the GS and the ADF test did not result in good power when considered for cases with one or two structural breaks. ${ }^{10}$ The ZA test, constructed to account for a single endogenous structural break in both the level and the trend slope, performed well in the case with one break, but experienced a large power loss for the case with two structural breaks. The LLC test did experience some power loss every time a break was added to the DGP. However, the power loss

\footnotetext{
${ }^{8}$ The Zivot and Andrews [1992] test has three variants: the ZA Model A test permits an change in the level of the series under the alternative hypothesis, the ZA Model B test allows for a change in the rate of growth, and the ZA Model $\mathrm{C}$ test admits both changes under the alternative. In the ZA testing procedure the time of the break is estimated rather than assumed as an exogenous phenomenon.

${ }^{9}$ In the event of sudden level shifts only, one could also consider a recently proposed range unit root (RUR) test by Aparicio et al. [2006] which is known to be robust to structural changes in the levels (shifts).

${ }^{10}$ In fairness, neither of these tests were designed to accommodate structural breaks. As noted above, they both performed quite well for the cases that they were designed to address.
} 


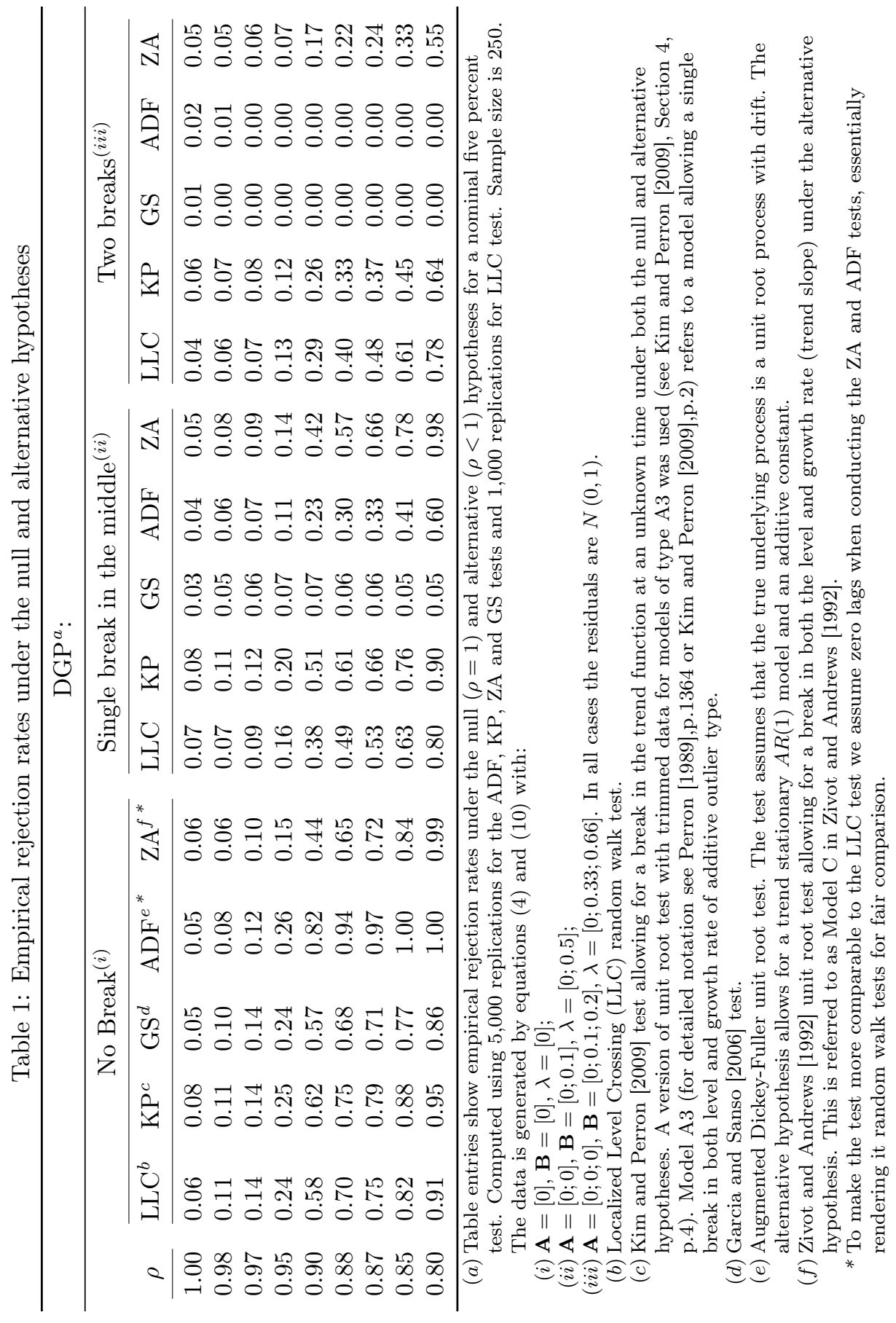


Table 2: Empirical crossing rates, $T^{-1 / 2} K_{T}^{(m)}(0)$, for different error distributions under the null hypothesis.

\begin{tabular}{|c|c|c|c|c|c|c|c|}
\hline Errors & $m=1$ & $m=2$ & $m=3$ & $m=5$ & $m=10$ & $m=25$ & $m=50$ \\
\hline & \multicolumn{7}{|c|}{ No Break ${ }^{(i)}$} \\
\hline$N(0,1)$ & 0.665 & 0.418 & 0.333 & 0.253 & 0.177 & 0.112 & 0.081 \\
\hline Student $-t_{5}$ & 0.665 & 0.416 & 0.328 & 0.247 & 0.171 & 0.107 & 0.077 \\
\hline$G A R C H(1,1)^{a}$ & 0.658 & 0.418 & 0.337 & 0.257 & 0.179 & 0.112 & 0.079 \\
\hline \multirow[t]{2}{*}{$E G A R C H(1,1)^{b}$} & 0.658 & 0.418 & 0.336 & 0.256 & 0.178 & 0.112 & 0.081 \\
\hline & \multicolumn{7}{|c|}{ Single break in the middle ${ }^{(i i)}$} \\
\hline$N(0,1)$ & 0.664 & 0.418 & 0.332 & 0.253 & 0.177 & 0.112 & 0.079 \\
\hline Student $-t_{5}$ & 0.665 & 0.416 & 0.328 & 0.247 & 0.171 & 0.107 & 0.076 \\
\hline$G A R C H(1,1)^{a}$ & 0.657 & 0.418 & 0.337 & 0.257 & 0.179 & 0.110 & 0.076 \\
\hline \multirow[t]{2}{*}{$E G A R C H(1,1)^{b}$} & 0.658 & 0.418 & 0.336 & 0.255 & 0.178 & 0.111 & 0.079 \\
\hline & \multicolumn{7}{|c|}{ Two breaks ${ }^{(i i i)}$} \\
\hline$N(0,1)$ & 0.665 & 0.418 & 0.332 & 0.253 & 0.176 & 0.109 & 0.070 \\
\hline Student $-t_{5}$ & 0.665 & 0.416 & 0.327 & 0.246 & 0.170 & 0.105 & 0.070 \\
\hline$G A R C H(1,1)^{a}$ & 0.657 & 0.418 & 0.336 & 0.257 & 0.178 & 0.106 & 0.066 \\
\hline $\operatorname{EGARCH}(1,1)^{b}$ & 0.658 & 0.418 & 0.336 & 0.255 & 0.177 & 0.109 & 0.072 \\
\hline Asymptotic $^{c}$ & 0.667 & 0.420 & 0.333 & 0.253 & 0.177 & 0.111 & 0.078 \\
\hline
\end{tabular}

Table entries show empirical crossing rates, defined by $T^{-1 / 2} K_{T}^{(m)}(0)=$

$T^{-1} \sum_{t=1}^{T-1} \mathbf{1}\left[x_{t}^{(m)} x_{t+1}^{(m)}<0\right]$, under the null $(\rho=1)$ hypothesis for different error distributions. Computed using 100,000 replications based on a sample size of $\mathrm{T}=250$. The data is generated by equations (4) and (10) with:

(i) $\mathbf{A}=[0], \mathbf{B}=[0], \lambda=[0]$;

(ii) $\mathbf{A}=[0 ; 0], \mathbf{B}=[0 ; 0.1], \lambda=[0 ; 0.5]$;

(iii) $\mathbf{A}=[0 ; 0 ; 0], \mathbf{B}=[0 ; 0.1 ; 0.2], \lambda=[0 ; 0.33 ; 0.66]$. In all cases the residuals are $N(0,1)$.

(a) GARCH(P,Q) model: $\sigma_{t}^{2}=k+\sum_{i=1}^{P} G_{i} \sigma_{t-i}^{2}+\sum_{j=1}^{Q} A_{j} \varepsilon_{t-j}^{2}$, where $\varepsilon_{t} \sim N(0,1)$. For our simulations we use $P=1, Q=1, k=0.2, G_{1}=0.3, A_{1}=0.5$.

(b) $\operatorname{EGARCH}(\mathrm{P}, \mathrm{Q})$ model:

$\log \sigma_{t}^{2}=k+\sum_{i=1}^{P} G_{i} \log \sigma_{t-i}^{2}+\sum_{j=1}^{Q} A_{j}\left[\frac{\left|\varepsilon_{t-j}\right|}{\sigma_{t-j}}-E\left\{\frac{\left|\varepsilon_{t-j}\right|}{\sigma_{t-j}}\right\}\right]+\sum_{j=1}^{Q} L_{j}\left(\frac{\varepsilon_{t-j}}{\sigma_{t-j}}\right)$, where $\varepsilon_{t} \sim N(0,1)$. For our simulations we use $P=1, Q=1, k=0.2, G_{1}=0.3$, $A_{1}=0.5, L_{1}=0.1$.

(c) Computed using equation (15). 
Table 3: Empirical crossing rates, $T^{-1 / 2} K_{T}^{(m)}(0)$, for different error distributions under the alternative hypothesis.

\begin{tabular}{|c|c|c|c|c|c|c|c|}
\hline Errors & $m=1$ & $m=2$ & $m=3$ & $m=5$ & $m=10$ & $m=25$ & $m=50$ \\
\hline & \multicolumn{7}{|c|}{ No Break ${ }^{(i)}$} \\
\hline$N(0,1)$ & 0.668 & 0.425 & 0.344 & 0.275 & 0.224 & 0.205 & 0.201 \\
\hline Student $-t_{5}$ & 0.669 & 0.423 & 0.339 & 0.269 & 0.217 & 0.198 & 0.194 \\
\hline$G A R C H(1,1)^{a}$ & 0.662 & 0.424 & 0.348 & 0.281 & 0.228 & 0.207 & 0.203 \\
\hline \multirow[t]{2}{*}{$E G A R C H(1,1)^{b}$} & 0.662 & 0.425 & 0.348 & 0.279 & 0.225 & 0.206 & 0.202 \\
\hline & \multicolumn{7}{|c|}{ Single break in the middle ${ }^{(i i)}$} \\
\hline$N(0,1)$ & 0.668 & 0.425 & 0.344 & 0.275 & 0.224 & 0.201 & 0.178 \\
\hline Student $-t_{5}$ & 0.669 & 0.423 & 0.340 & 0.269 & 0.216 & 0.195 & 0.178 \\
\hline$G A R C H(1,1)^{a}$ & 0.662 & 0.424 & 0.349 & 0.280 & 0.227 & 0.202 & 0.170 \\
\hline \multirow[t]{2}{*}{$E G A R C H(1,1)^{b}$} & 0.662 & 0.425 & 0.348 & 0.278 & 0.225 & 0.203 & 0.183 \\
\hline & \multicolumn{7}{|c|}{ Two breaks ${ }^{(i i i)}$} \\
\hline$N(0,1)$ & 0.668 & 0.425 & 0.344 & 0.275 & 0.222 & 0.187 & 0.112 \\
\hline Student $-t_{5}$ & 0.669 & 0.423 & 0.339 & 0.268 & 0.215 & 0.185 & 0.128 \\
\hline$G A R C H(1,1)^{a}$ & 0.661 & 0.425 & 0.348 & 0.280 & 0.224 & 0.181 & 0.097 \\
\hline $\operatorname{EGARCH}(1,1)^{b}$ & 0.662 & 0.425 & 0.348 & 0.278 & 0.224 & 0.191 & 0.125 \\
\hline Asymptotic ${ }^{c}$ & 0.670 & 0.426 & 0.345 & 0.275 & 0.223 & 0.205 & 0.205 \\
\hline
\end{tabular}

Table entries show empirical crossing rates, defined by $T^{-1 / 2} K_{T}^{(m)}(0)=$

$T^{-1} \sum_{t=1}^{T-1} \mathbf{1}\left[x_{t}^{(m)} x_{t+1}^{(m)}<0\right]$, under the alternative $(\rho=0.8)$ hypothesis for different error distributions. Computed using 100,000 replications based on a sample size of $\mathrm{T}=250$. The data is generated by equations (4) and (10) with:

(i) $\mathbf{A}=[0], \mathbf{B}=[0], \lambda=[0]$;

(ii) $\mathbf{A}=[0 ; 0], \mathbf{B}=[0 ; 0.1], \lambda=[0 ; 0.5]$;

(iii) $\mathbf{A}=[0 ; 0 ; 0], \mathbf{B}=[0 ; 0.1 ; 0.2], \lambda=[0 ; 0.33 ; 0.66]$. In all cases the residuals are $N(0,1)$.

(a) GARCH(P,Q) model: $\sigma_{t}^{2}=k+\sum_{i=1}^{P} G_{i} \sigma_{t-i}^{2}+\sum_{j=1}^{Q} A_{j} \varepsilon_{t-j}^{2}$, where $\varepsilon_{t} \sim N(0,1)$. For our simulations we use $P=1, Q=1, k=0.2, G_{1}=0.3, A_{1}=0.5$.

(b) $\operatorname{EGARCH}(\mathrm{P}, \mathrm{Q})$ model:

$\log \sigma_{t}^{2}=k+\sum_{i=1}^{P} G_{i} \log \sigma_{t-i}^{2}+\sum_{j=1}^{Q} A_{j}\left[\frac{\left|\varepsilon_{t-j}\right|}{\sigma_{t-j}}-E\left\{\frac{\left|\varepsilon_{t-j}\right|}{\sigma_{t-j}}\right\}\right]+\sum_{j=1}^{Q} L_{j}\left(\frac{\varepsilon_{t-j}}{\sigma_{t-j}}\right)$, where $\varepsilon_{t} \sim N(0,1)$. For our simulations we use $P=1, Q=1, k=0.2, G_{1}=0.3$, $A_{1}=0.5, L_{1}=0.1$.

(c) Computed using equation (15). 
Table 4: Empirical rejection rates under the null and alternative hypotheses for different error distributions

\begin{tabular}{|c|c|c|c|c|c|c|}
\hline \multicolumn{7}{|c|}{$\mathrm{DGP}^{a}$ : } \\
\hline \multirow[b]{2}{*}{$\rho$} & \multicolumn{3}{|c|}{ No Break ${ }^{(i)}$} & \multicolumn{3}{|c|}{ Single break in the middle $\mathrm{e}^{(i i)}$} \\
\hline & $\mathrm{LLC}^{b}$ & $\mathrm{GS}^{c}$ & $\mathrm{ADF}^{d}$ & LLC & GS & $\mathrm{ADF}^{e}$ \\
\hline \multicolumn{7}{|c|}{$N(0,1)$ innovations } \\
\hline 1.00 & 0.06 & 0.05 & 0.06 & 0.07 & 0.03 & 0.05 \\
\hline 0.85 & 0.85 & 0.77 & 0.96 & 0.634 & 0.05 & 0.27 \\
\hline \multicolumn{7}{|c|}{ Student $t_{5}$ innovations } \\
\hline 1.00 & 0.06 & 0.06 & 0.054 & 0.06 & 0.03 & 0.06 \\
\hline 0.85 & 0.76 & 0.77 & 0.96 & 0.68 & 0.05 & 0.28 \\
\hline \multicolumn{7}{|c|}{$G A R C H(1,1)$ innovations $^{f}$} \\
\hline 1.00 & 0.056 & 0.06 & 0.048 & 0.066 & 0.02 & 0.064 \\
\hline 0.85 & 0.774 & 0.75 & 0.951 & 0.574 & 0.04 & 0.147 \\
\hline \multicolumn{7}{|c|}{$E G A R C H(1,1)$ innovations $^{g}$} \\
\hline 1.00 & 0.05 & 0.053 & 0.046 & 0.07 & 0.01 & 0.075 \\
\hline 0.85 & 0.712 & 0.74 & 0.954 & 0.65 & 0.03 & 0.446 \\
\hline \multirow{2}{*}{\multicolumn{7}{|c|}{$\begin{array}{l}\text { Table entries show empirical rejection rates under the null } \\
(\rho=1) \text { and alternative }(\rho=0.85) \text { hypotheses for a nominal five } \\
\text { percent test. Computed using } 5,000 \text { replications for the ADF } \\
\text { and GS tests and } 1,000 \text { replications for LLC test. Sample size is } \\
250 . \text { The data is generated by equations }(4) \text { and }(10) \text { with: } \\
\mathbf{A}=[0], \mathbf{B}=[0], \lambda=[0] ; \\
\mathbf{A}=[0 ; 0], \mathbf{B}=[0 ; 0.1], \lambda=[0 ; 0.5] \text {; } \\
\text { Localized Level Crossing (LLC) random walk test. } \\
\text { Garcia and Sanso [2006] test. } \\
\text { Augmented Dickey-Fuller test with no trend or constant. } \\
\text { Augmented Dickey-Fuller test with trend and no constant. } \\
\text { GARCH }(\mathrm{P}, \mathrm{Q}) \text { model: } \sigma_{t}^{2}=k+\sum_{i=1}^{P} G_{i} \sigma_{t-i}^{2}+\sum_{j=1}^{Q} A_{j} \varepsilon_{t-j}^{2}, \\
\text { where } \varepsilon_{t} \sim N(0,1) \text { For our simulations we use } P=1, Q=1, \\
k=0.2, G_{1}=0.3, A_{1}=0.5 \\
\text { EGARCH(P,Q) model: } \log \sigma_{t}^{2}=k+\sum_{i=1}^{P} G_{i} \log \sigma_{t-i}^{2}+\end{array}$}} \\
\hline & & & & & & \\
\hline \multicolumn{2}{|c|}{$\sum_{j=1}^{Q} A_{j}$} & \multicolumn{2}{|c|}{ 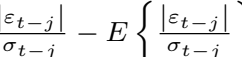 } & \multicolumn{3}{|c|}{$+\sum_{j=1}^{Q} L_{j}\left(\frac{\varepsilon_{t-j}}{\sigma_{t-j}}\right)$, where } \\
\hline
\end{tabular}



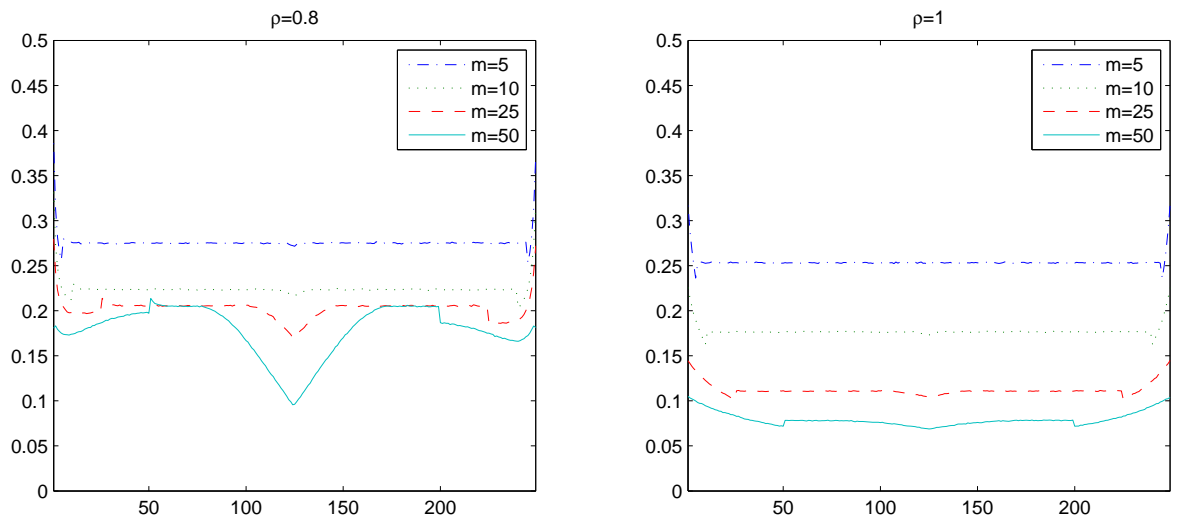

Figure 8: Average crossing rates for series with a single break in the middle for $m=5,15,25$ and 50 for $\rho=0.8$ (left panel) and $\rho=1$ (right panel). The left panel in the figure shows that the crossing rate deteriorates faster for larger values of $m$ both due to the presence of the break and due to linear extrapolation of the boundary points defined in (2) towards the beginning and end of the sample. The deterioration is mild for all four values of $m$ under the null hypothesis (right panel). Simulated using $T=250$, Gaussian errors, $\mathbf{A}=[0 ; 0], \mathbf{B}=[0 ; 0.1]$ and $\lambda=[0 ; 0.5]$. Computed using 100,000 simulations.

was smaller than that of the ZA procedure when comparing one- and two-break scenarios. The LLC also maintained a relatively constant size across all three cases. Thus, the LLC random walk test proposed in this paper offers an improvement over commonly used methods in moderately sized samples for cases with two or more breaks. Likewise, it may be useful in cases for which the number of breaks is unknown.

The preceding simulations employed i.i.d. Gaussian errors. It is also important to assess the finite sample accuracy of the test under distributions with fatter tails, as this is a common feature of many economic and financial time series. Similarly, in deriving the null asymptotics we required both higher moment independence $(\mathscr{A} .1)$ and elliptical symmetry ( $\mathscr{A} .3)$. We would also like to study the behavior of the test in practical situations where these assumptions may be violated. Of particular relevance to financial data are models with conditionally heteroskedastic errors.

Before turning to the test itself, in Table 2 we first examine the null crossing rates, defined by $T^{-1 / 2} K_{T}^{(m)}(0)=T^{-1} \sum_{t=1}^{T-1} \mathbf{1}\left[x_{t}^{(m)} x_{t+1}^{(m)}<0\right]$, on which the LLC test is based. We compare the null crossing rates using standard normal, Student- $t$ (with 5 degrees of freedom), GARCH and EGARCH innovations for different values of $m$. For all values of $m$ the simulated null rejection rates for the standard normal and Student- $t(d f=5)$ innovation distributions are nearly indistinguishable. Since both belong to the family of elliptically symmetric distributions specified in (A .3), this is expected from equation (15). More interestingly, crossing rates for both the GARCH and EGARCH models, which lie outside this family, are still quite similar. Therefore, one might consider applying the LLC test to data with conditionally heteroskedastic innovations, even though the test is derived under the assumption of conditional homoskedasticity.

Figure 8 shows how the average crossing rates are affected by the presence of a single break in the middle of a series. Both the presence of the break and the need for linear extrapolation of the boundary points result in underestimation of the number of crossings. For greater values of the localization parameter, $m$, the underestimation of the number of crossings is more severe and hence the larger is the power loss. Our simulations show that this is especially true for lower values of autoregressive parameter $\rho$. When $\rho=1$ (right panel), the deterioration of average 
crossing rates due to the presence of the break is mild for all four values of $m$. The location of the break in this case becomes less important.

To provide additional intuition, in Table 3 we examine the average crossing rates for an alternative hypothesis when $\rho=0.8$. The table has three panels showing three different cases: (i) a case with no break, (ii) single break and (iii) two breaks. As expected, average crossing rates across the three cases are relatively stable for values of $m=1,2,3$ and 5 but deteriorate for larger values of $m$, especially in the case when $m=50$.

In Table 4 we address this issue more directly by studying the null rejection rates themselves, shown in the top row of each panel. We consider the same four innovation distributions in both a no break and single break cases and provide a comparison of the LLC test to two benchmarks: the GS and ADF tests. As the table shows, the null rejection rates of the LLC test remain close to the nominal size under all four innovation distributions and compare favorably with the several of the benchmark cases.

It is also interesting to assess the sensitivity of the test power under these different error distributions. The second row of each panel in Table 4 shows the empirical rates under the alternative hypothesis when $\rho=0.85$. In the no break case the LLC test suffers a modest decline in power under the three alternate error distributions relative to the normal distribution, whereas the power of the GS and ADF tests appears stable across these distributions. On the other hand, the power of LLC test is more stable across error distributions in the single break case and even rises modestly in the case of student- $t_{5}$ errors. The power of both the GS and ADF test continues to be poor across all four error distributions in the single break case and the power of the ADF test also appears somewhat unstable across error distributions.

\section{Empirical Application}

\subsection{Nelson-Plosser macroeconomic data set}

Nelson and Plosser [1982]'s finding that the unit root could not be rejected in most macroeconomic time series has inspired scholars to re-examine the unit root hypothesis and devise tests with greater power against plausible stationary alternatives, including tests accounting for structural breaks. We apply our proposed test procedure to the Nelson-Plosser data set and compare the results to some representative existing unit root test (See Table 5.).

In addition to the results of the LLC test, we include the results based on the ADF test, the Garcia and Sanso [2006]'s test (GS), the Zivot and Andrews [1992]'s test (ZA), the Park and Fuller [1995|'s weighted symmetric DF test (PF-WS), the Elliott et al. [1996]'s GLS test (ERS-GLS), Perron [1989]'s original test (P) and Kim and Perron [2009] test (KP). The LLC and GS both rely on the level crossings of a process. The LLC, ZA, PF-WS, ERS-GLS and KP tests allow for endogenous breaks while the $\mathrm{P}$ test allows for exogenously determined breaks.

The LLC test rejected the null hypothesis of a random walk for two series at the five percent significance level: the unemployment rate and industrial production. This result is consistent with findings by Elliott et al. [1996], Zivot and Andrews [1992] and Perron [1989]. In comparison to Perron [1989] and Park and Fuller [1995], the LLC test failed to reject an additional five series (namely real GNP, per-capita real GNP, nominal wages, real wages and money stock). The ZA test, allowing for a single endogenous break and being the closest alternative to the LLC test, rejects the unit root hypothesis for two additional series, real GNP and nominal GNP. Perron [1989]'s original test rejects the unit root null for most of the series, but unlike the LLC and ZA 


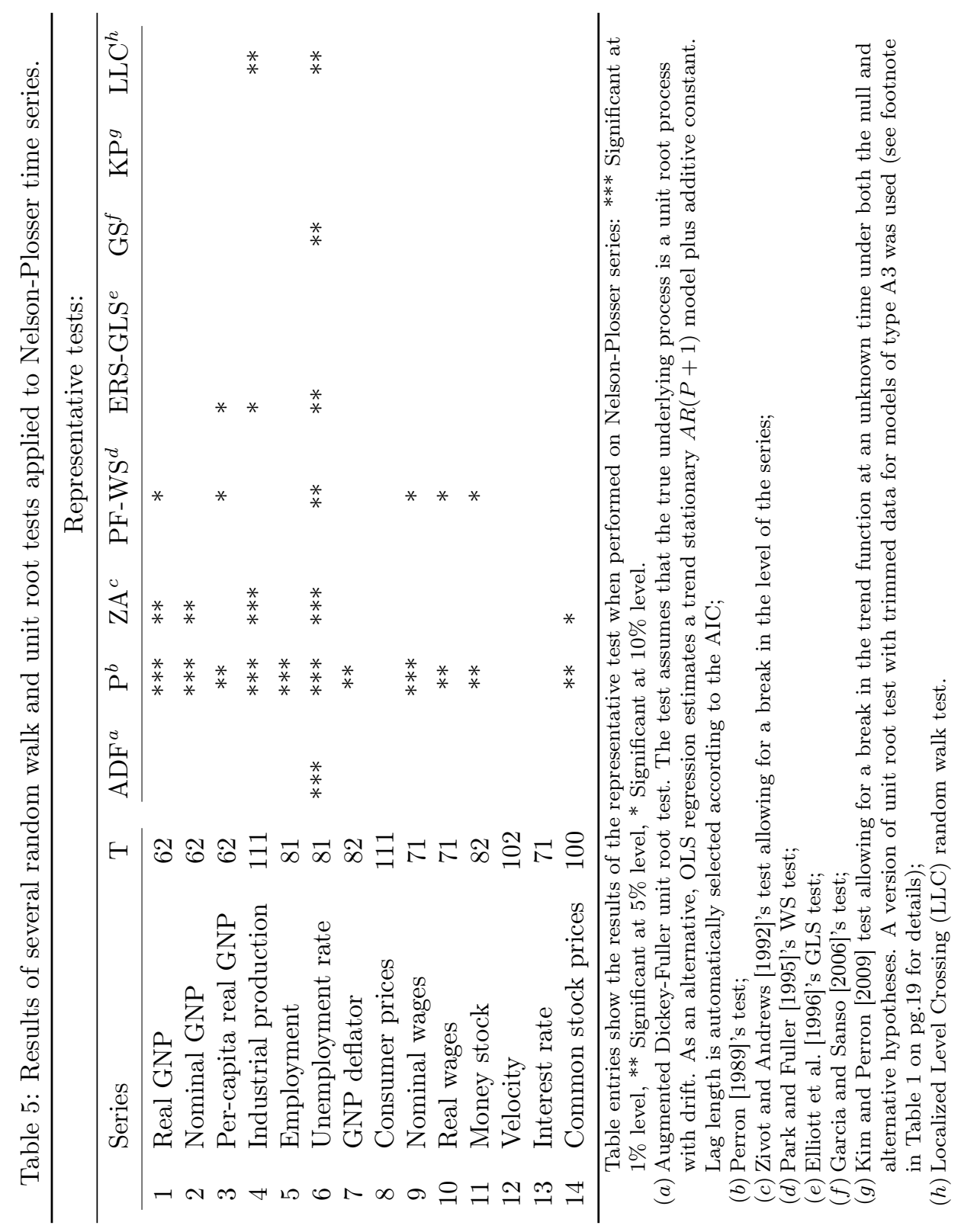


tests it assumes a known a priori break location. However, it should be noted, that the LLC testing procedure, which is designed as a random walk (not a unit root) test, does not account for serial correlations, often present in macroeconomic data. The KP test failed to reject the null hypothesis for all fourteen series, surprisingly even for unemployment rate. This might be partially due to the fact that the KP procedure relies on the assumption that the structural break must exist in the data. Kim and Perron [2009] mention that this assumption is an important limitation (e.g. Perron [1989]'s original test (P) does not require a break to occur, it only allow for such a possibility). Thus, in the absence of a structural break in the data the KP test ${ }^{11}$ might not be reliable.

\subsection{Application: Inflation and Interest Rates in Canada}

The relationship between the inflation and the interest rates has always been a topic of interest in financial economics. The well known Fisher [1930] hypothesis posits a positive one-to-one relationship between the inflation and nominal interest rate. A large literature tests this hypothesis using cointegration methods under the assumption that the nominal interest and inflation rates are nonstationary (see Mishkin [1992], Owen [1993] and Evans and Lewis [1995]). However, evidence of I(1) behavior could be due to the presence of structural breaks, in which case cointegration tests might not be the most appropriate method to test the Fisher hypothesis.

Atkins and Chan [2004], using methodology of Zivot and Andrews [1992], Lumsdaine and Papell [1997] and Banerjee et al. [1992], find evidence that the 90-day Treasury Bill rate and the inflation rate in Canada are stationary series with two breaks. Using the Zivot and Andrews [1992] procedure, which only allows for a single break, the authors find breaks in the inflation and interest rates at 1980Q3 and 1972Q3 respectively. Using the Lumsdaine and Papell [1997] procedure, and allowing for up to two breaks in both intercept and trend (model CC in Lumsdaine and Papell [1997]), they find two breaks in inflation at 1972Q3 and 1982Q2 and in the interest rate at 1980Q3 and 1988Q1.

In our empirical analysis, we employ the Bank of Canada 90-day Treasury bill rate as a proxy for the nominal interest rate. We use CPI on all items for the year 2000 basket as a proxy for calculating nominal inflation. The data consists of quarterly observations for the period of 1954Q1-2007Q2 corresponding to a sample size of 216.

Figure 9 traces the path of Canadian inflation for the period of 1954Q1-2007Q2, showing what are often referred to as the twin peaks of Canadian inflation, when inflation rose to over 14 per cent in 1973 and to almost 13 per cent in 1979. The chart also shows the sharp decline in inflation during the early 1980s, from over 12 per cent to 4 per cent, the modest increase from 4 per cent to 6 per cent over the balance of the 1980s, and then the reduction from just over 6 per cent in 1990 to roughly 2 per cent over the following few years. From 1992 to 2007, the rate of CPI inflation in Canada has fluctuated around 2 per cent. Figure 10 shows nominal interest rate with a peak value of just above 20 per cent in the early '80s.

Using the LLC procedure, we conduct a random walk test on the interest and inflation rates that is robust to these potential breaks. The results are displayed in Table 6 . The top panel provides the results for the LLC test, while the bottom panel provides a comparison to several other tests. As seen from the table, using the LLC test, we reject the null hypothesis of a random walk for both the inflation and interest rates. In contrast, the ADF, ZA Model B and GS tests fail to reject a unit root in either variable. The ZA Model C test rejects the unit root hypothesis for the interest rate but fails to reject it for the inflation rate (perhaps due to the presence of the third

\footnotetext{
${ }^{11}$ In particular, we refer to Kim and Perron [2009]'s unit root test with trimmed data, Section 4, pp.4-5.
} 


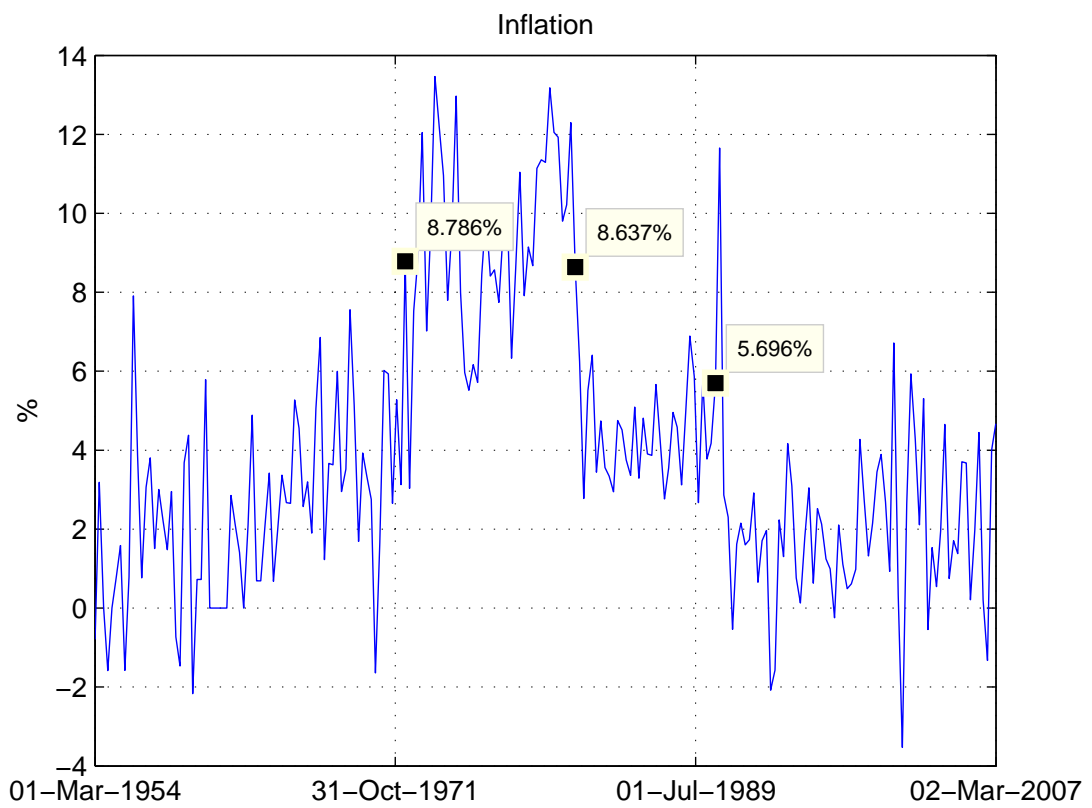

Figure 9: Inflation rate (Source: CANSIM, Consumer price index (CPI), 2001 basket content, 1992=100, Canada, quarterly, 1954Q1-2007Q2).

break in that series).

In selecting the localization parameter, $m^{*}$, we estimated a proximate model in which the number of breaks was selected by the Bai and Perron [1998] procedure. Interestingly, it identified three breaks in the inflation rate, corresponding to 1972Q3, 1982Q3 and 1990Q4. The first two breaks are also detected by Lumsdaine and Papell [1997]'s procedure. The location of these breaks is consistent with the downturn in the Canadian economy in the early 1980s and the Bank of Canada's change in it's monetary policy in late 1979. The break in the early 1970's could, perhaps, be attributed to the break down of the Bretton Woods system. The third break corresponds to a sharp reduction in inflation corresponding to the introduction of inflation targeting by the Bank of Canada in 1991. Two breaks were found in the interest rate at 1981Q3 and 1990Q4. These breaks are marked in figures 9 and 10. The values of $m$ selected by the LLC procedure were 6 and 31 for the inflation and interest rate respectively. This is consistent with our conjecture in Section 3 that the LLC procedure would tend to select a lower value of $m$ for series with a higher number of estimated breaks.

\section{Concluding Remarks}

In this paper we proposed a new localized level crossing (LLC) random walk test that is robust to an unspecified number, location, and magnitude of breaks in both the level and slope of the deterministic trend. An informal method was proposed to select the localization parameter in order to maximize the bootstrapped power by resampling the residuals from an estimated proximate model. A second outer-bootstrap, in which the localization parameter search was repeated on each bootstrap replicate, was proposed in an attempt to improve the test size using critical values that reflect the pre-selection inherent in the inner bootstrap. Our simulations confirmed 
Table 6: Results of the LLC test applied to Canadian data

\begin{tabular}{|c|c|c|}
\hline & Inflation & Interest Rate \\
\hline & \multicolumn{2}{|c|}{ LLC test $^{a}$} \\
\hline & \multicolumn{2}{|c|}{ Test results } \\
\hline test statistic $\left(t^{\left(m^{*}\right)}\right)$ & $7.2673^{\psi}$ & $2.0327^{\psi}$ \\
\hline critical value $\left(t_{.95}^{\left(m^{*}\right)}\right)$ & 2.2801 & 1.8756 \\
\hline $\mathrm{p}$-value & 0.000 & 0.044 \\
\hline \multirow[t]{2}{*}{ Localization parameter $\left(m^{*}\right)$} & 6 & 31 \\
\hline & \multicolumn{2}{|c|}{ Proximate model estimates $^{b}$} \\
\hline Estimated break dates $(\hat{\lambda})$ & 1972Q3, 1982Q3, 1990Q4 & 1981Q3, 1990Q4 \\
\hline \multirow[t]{2}{*}{ Autoregressive coefficient $(\hat{\rho})$} & 0.1260 & 0.8383 \\
\hline & \multicolumn{2}{|c|}{ Comparison results from other tests } \\
\hline Selected lag length $(\mathrm{AIC})^{c}$ & 3 & 7 \\
\hline $\mathrm{ADF}_{\text {statistic }}{ }^{d}$ & -2.3450 & -1.7414 \\
\hline ZA Model B statistic ${ }^{e}$ & -3.54 & -4.13 \\
\hline ZA Model C statistic ${ }^{e}$ & -5.00 & $-5.15^{\psi}$ \\
\hline GS statistic $\left(\eta^{(G S)}\right)^{f}$ & 1.6367 & 1.1412 \\
\hline KP statistic ${ }^{g}$ & -4.1218 & -2.8287 \\
\hline KP estimated break dates & 1982Q1 & 1981Q3 \\
\hline RUR statistic ${ }^{h}$ & $0.6167^{\psi}$ & 1.7773 \\
\hline
\end{tabular}

$\psi$ denotes a rejection at the 5 percent nominal level.

(a) Within the LLC test procedure we use 1,000 replications for the inner bootstrap used to select $m^{*}$ and 1,000 simulations for the outer bootstrap procedure used for selecting critical values, $t_{95}^{\left(m^{*}\right)}$.

(b) We use the method of Bai and Perron [1998] to estimate the proximate model in (20) that we employ in the inner residual bootstrap used to select $m^{*}$. The proximate model used in the outer residual bootstrap used to select the critical value $t_{95}^{\left(m^{*}\right)}$ employs the same value of $\hat{\lambda}$ but sets $\rho=1$ to impose the null hypothesis.

(c) The lag-length employed in the ADF and ZA tests. (The GS test does not require a lag-length.) The lag-length was selected by the Akaike information criterion (AIC) in the model used for the ADF test, which allows for an intercept and trend but not structural breaks.

(d) The $5 \%$ critical value is -3.4335 .

(e) The $5 \%$ critical values for the ZA Model B test is -4.42 ; for the ZA Model C test it is -5.08, (Zivot and Andrews [1992], Table 4, pp.256-257). The ZA Model B permits a single break in trend growth rate; the ZA Model C test permits a single break in trend growth rate and/or trend level.

$(f)$ The $5 \%$ critical value for the Garcia and Sanso [2006] GS test statistic, $\eta^{(G S)}$ defined in (7), is 2.45 .

(g) Kim and Perron [2009] test allowing for a break in the trend function at an unknown time under both the null and alternative hypotheses. A version of unit root test with trimmed data for models of type A3 was used (see Kim and Perron [2009], Section 4, p.4). Model A3 (for detailed notation see Perron [1989],p.1364 or Kim and Perron [2009],p.2) refers to a model allowing a single break in both level and growth rate of additive outlier type. The $5 \%$ critical value for the test is -4.24 (Perron [1989], Table VI.B, p.1377)

(h) Aparicio et al. [2006]'s Range Unit Root (RUR) test. The test is robust to structural changes in the trend level only. The $5 \%$ critical value for the test is 1.1 (Aparicio et al. [2006], Table 1, p.549). 


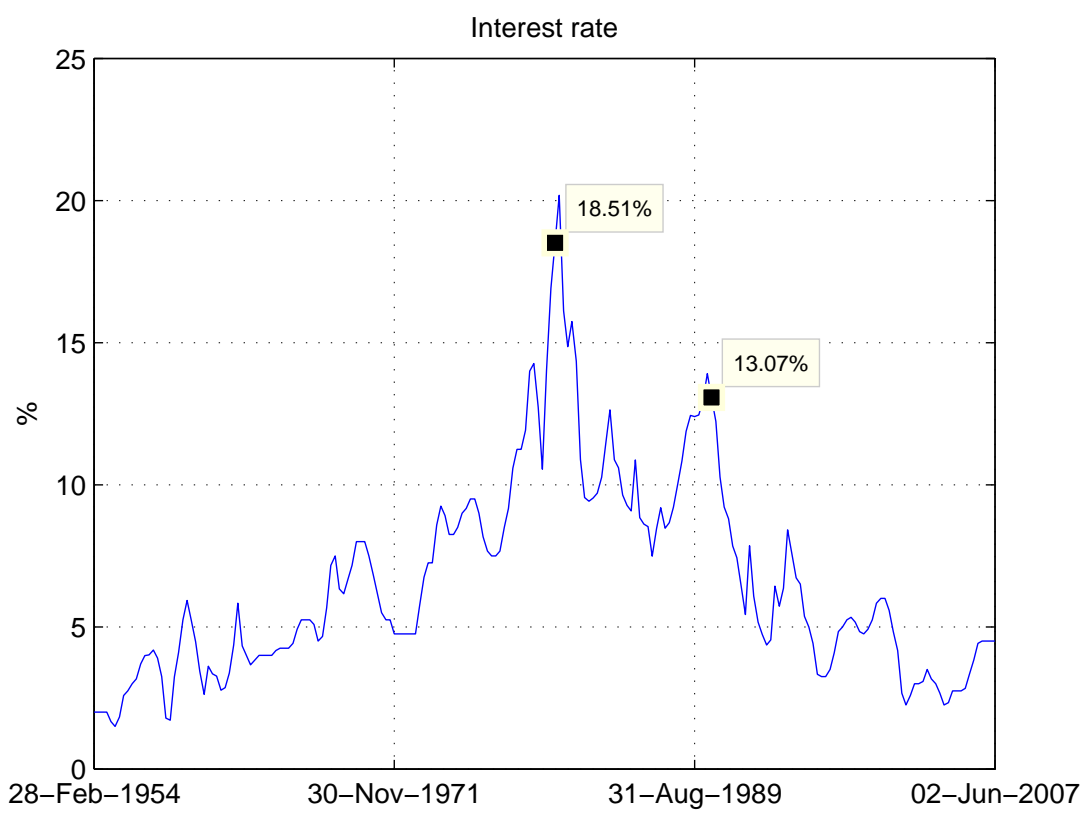

Figure 10: Interest rate (Source: CANSIM, Bank of Canada Rate, quarterly, 1954Q1-2007Q2).

that this procedure works well both in controlling for size and in producing good and relatively stable power across models with zero, one or two breaks. Applying our test to Canadian interest rate and inflation data, we are able to reject a random walk hypothesis in both series. This may have implications for tests of the Fisher hypothesis, which are often conducted using cointegration methods.

The selection of tuning parameters, including bandwidths and lag-lengths, is ubiquitous in econometrics. In a testing context, if such tuning parameters are pre-selected to improve test power, this may result in size distortion. Thus computational methods similar to the type informally proposed here may have broader applicability in other contexts. Establishing their theoretical properties could therefore provide an interesting direction for future research. 


\section{A Appendix}

\section{A.1 Proofs of Propositions and Lemmas}

Proof of Proposition 1 For $m=T-1$ we have:

$$
\begin{aligned}
x_{t-(T-1)} & =x_{1}+c(t-(T-1)) \\
x_{t+T-1} & =x_{T}+c(t-1)
\end{aligned}
$$

and therefore

$$
\begin{aligned}
x_{t}^{(T-1)} & =x_{t}-x_{t-(T-1)}-\frac{x_{t+T-1}-x_{t-(T-1)}}{2} \\
& =x_{t}-x_{1}-c(t-(T-1))-\frac{x_{T}+c(t-1)-x_{1}-c(t-(T-1))}{2} \\
& =x_{t}-x_{1}-c t-\frac{x_{T}-x_{1}-c T}{2}=x_{t}-x_{1}-c t .
\end{aligned}
$$

Proof of Proposition 2 First, consider the case in which $|\rho|<1$. Using the moving average representation for $u_{t}$ we have

$$
u_{t-m}=\sum_{j=0}^{\infty} \rho^{j} \varepsilon_{t-m-j}
$$

Then, by recursive backward substitution:

$$
u_{t}=\rho^{m} u_{t-m}+\sum_{j=0}^{m-1} \rho^{j} \varepsilon_{t-j} \quad \text { and } \quad u_{t+m}=\rho^{2 m} u_{t-m}+\sum_{j=0}^{2 m-1} \rho^{j} \varepsilon_{t+m-j} .
$$

This allows us to express $u_{t}^{(m)}$ as

$$
\begin{aligned}
u_{t}^{(m)}= & u_{t}-\frac{1}{2} u_{t-m}-\frac{1}{2} u_{t+m} \\
= & -\frac{1}{2}\left(\rho^{m}-1\right)^{2} \sum_{j=0}^{\infty} \rho^{j} \varepsilon_{t-m-j}+\left(1-\frac{1}{2} \rho^{m}\right) \sum_{j=0}^{m-1} \rho^{j} \varepsilon_{t-j} \\
& -\frac{1}{2} \sum_{j=0}^{m-1} \rho^{j} \varepsilon_{t+m-j}
\end{aligned}
$$

To simplify notation define $a=-\frac{1}{2}\left(\rho^{m}-1\right)^{2} ; b=1-\frac{1}{2} \rho^{m} ; c=-\frac{1}{2}$, then we have the following:

$$
u_{t}^{(m)}=a \sum_{j=0}^{\infty} \rho^{j} \varepsilon_{t-m-j}+b \sum_{j=0}^{m-1} \rho^{j} \varepsilon_{t-j}+c \sum_{j=0}^{m-1} \rho^{j} \varepsilon_{t+m-j} .
$$

Then, using the conditions in (14) and noting that

$$
\begin{aligned}
\mathbb{E}\left[\sum_{j=0}^{\infty} \rho^{j} \varepsilon_{t-m-j} \sum_{j=0}^{m-1} \rho^{j} \varepsilon_{t+h-j}\right] & =\mathbb{E}\left[\sum_{j=0}^{\infty} \rho^{j} \varepsilon_{t-m-j} \sum_{j=0}^{m-1} \rho^{j} \varepsilon_{t+m+h-j}\right] \\
& =\mathbb{E}\left[\sum_{j=0}^{m-1} \rho^{j} \varepsilon_{t-j} \sum_{j=0}^{m-1} \rho^{j} \varepsilon_{t+m+h-j}\right]=0, \quad \forall h
\end{aligned}
$$


we have the following:

$$
\begin{aligned}
\gamma_{h}^{(m)} & \equiv E\left(u_{t}^{(m)} u_{t+h}^{(m)}\right)=a^{2} \mathbb{E}\left[\sum_{j=0}^{\infty} \rho^{j} \varepsilon_{t-m-j} \sum_{j=0}^{\infty} \rho^{j} \varepsilon_{t-m+h-j}\right] \\
& +a b \mathbb{E}\left[\sum_{j=0}^{m-1} \rho^{j} \varepsilon_{t-j} \sum_{j=0}^{\infty} \rho^{j} \varepsilon_{t-m+h-j}\right]+b^{2} \mathbb{E}\left[\sum_{j=0}^{m-1} \rho^{j} \varepsilon_{t-j} \sum_{j=0}^{m-1} \rho^{j} \varepsilon_{t+h-j}\right] \\
& +a c \mathbb{E}\left[\sum_{j=0}^{m-1} \rho^{j} \varepsilon_{t+m-j} \sum_{j=0}^{\infty} \rho^{j} \varepsilon_{t-m+h-j}\right]+b c \mathbb{E}\left[\sum_{j=0}^{m-1} \rho^{j} \varepsilon_{t+m-j} \sum_{j=0}^{m-1} \rho^{j} \varepsilon_{t+h-j}\right] \\
& +c^{2} \mathbb{E}\left[\sum_{j=0}^{m-1} \rho^{j} \varepsilon_{t+m-j} \sum_{j=0}^{m-1} \rho^{j} \varepsilon_{t+m+h-j}\right] .
\end{aligned}
$$

The summands in the expression above can be solved for as follows:

$$
\begin{aligned}
& \mathbb{E}\left[\sum_{j=0}^{\infty} \rho^{j} \varepsilon_{t-m-j} \sum_{j=0}^{\infty} \rho^{j} \varepsilon_{t-m+h-j}\right]=\sigma_{\varepsilon}^{2} \sum_{j=0}^{\infty} \rho^{j} \rho^{j+h} \\
& \mathbb{E}\left[\sum_{j=0}^{m-1} \rho^{j} \varepsilon_{t-j} \sum_{j=0}^{\infty} \rho^{j} \varepsilon_{t-m+h-j}\right]= \begin{cases}0 & h=0 \\
\sigma_{\varepsilon}^{2} \sum_{j=0}^{h-1} \rho^{j} \rho^{m-h+j} & \forall h=1 . . m \\
\sigma_{\varepsilon}^{2} \sum_{j=0}^{m-1} \rho^{j} \rho^{h-m+j} & \forall h>m\end{cases} \\
& \mathbb{E}\left[\sum_{j=0}^{m-1} \rho^{j} \varepsilon_{t-j} \sum_{j=0}^{m-1} \rho^{j} \varepsilon_{t+h-j}\right]= \begin{cases}\sigma_{\varepsilon}^{2} \sum_{j=0}^{m-1-h} \rho^{j} \rho^{j+h} & \forall h=0 . . m-1 \\
0 & \forall h \geq m\end{cases} \\
& \mathbb{E}\left[\sum_{j=0}^{m-1} \rho^{j} \varepsilon_{t+m-j} \sum_{j=0}^{\infty} \rho^{j} \varepsilon_{t-m+h-j}\right]= \begin{cases}0 & \forall h=0 . . m-1 \\
\sigma_{\varepsilon}^{2} \sum_{j=0}^{h-m-1} \rho^{j} \rho^{2 m-h+j} & \forall h=m . .2 m-1 \\
\sigma_{\varepsilon}^{2} \sum_{j=0}^{m-1} \rho^{j} \rho^{h-2 m+j} & \forall h \geq 2 m\end{cases} \\
& \mathbb{E}\left[\sum_{j=0}^{m-1} \rho^{j} \varepsilon_{t+m-j} \sum_{j=0}^{m-1} \rho^{j} \varepsilon_{t+h-j}\right]= \begin{cases}0 & h=0 \\
\sigma_{\varepsilon}^{2} \sum_{j=0}^{h-1} \rho^{j} \rho^{m-h+j} & h=1 . . m \\
\sigma_{\varepsilon}^{2} \sum_{j=0}^{h-m-1} \rho^{j} \rho^{h-m+j} & \forall h=m+1 . .2 m-1 \\
0 & \forall h \geq 2 m\end{cases} \\
& \mathbb{E}\left[\sum_{j=0}^{m-1} \rho^{j} \varepsilon_{t+m-j} \sum_{j=0}^{m-1} \rho^{j} \varepsilon_{t+m+h-j}\right]= \begin{cases}\sigma_{\varepsilon}^{2} \sum_{j=0}^{m-1-h} \rho^{j} \rho^{j+h} & \forall h=0 . . m-1 \\
0 & \forall h \geq m .\end{cases}
\end{aligned}
$$

The autocorrelation function can then be shown to equal:

$$
r_{u, h}^{(m)} \equiv \frac{\gamma_{h}^{(m)}}{\gamma_{0}^{(m)}}= \begin{cases}1 & h=0 \\ \frac{\rho^{h}}{2}+\frac{3 \rho^{h}+\rho^{2 m-h}-4 \rho^{m-h}}{2\left(1-\rho^{m}\right)\left(3-\rho^{m}\right)} & h=1 . . m \\ \frac{\rho^{h}}{2}+\frac{3 \rho^{h}-4 \rho^{h-m}+\rho^{2 m-h}\left(\rho^{2 m}-2 \rho^{m}+1\right)+2 \rho^{3 h-3 m}-\rho^{3 h-2 m}}{2\left(1-\rho^{m}\right)\left(3-\rho^{m}\right)} & h=m+1 . .2 m-1 \\ \frac{\rho^{h}}{2}+\frac{3 \rho^{h}-4 \rho^{m-h}+\rho^{h-m}}{2\left(1-\rho^{m}\right)\left(3-\rho^{m}\right)} & h \geq 2 m\end{cases}
$$


Next, consider the case when $\rho=1$. Again using the the moving average representation and recursive substitution, we have

$$
\begin{aligned}
u_{t-m} & =\sum_{j=0}^{\infty} \varepsilon_{t-m-j}, \quad u_{t}=u_{t-m}+\sum_{j=0}^{m-1} \varepsilon_{t-j}, \quad u_{t+m}=u_{t-m}+\sum_{j=0}^{2 m-1} \varepsilon_{t+m-j} \\
u_{t}^{(m)} & =u_{t}-\frac{1}{2} u_{t-m}-\frac{1}{2} u_{t+m}=\frac{1}{2}\left(\sum_{j=0}^{m-1} \varepsilon_{t-j}-\sum_{j=0}^{m-1} \varepsilon_{t+m-j}\right) .
\end{aligned}
$$

Using (14) we find

$$
\begin{aligned}
\gamma_{h}^{(m)} \equiv E\left(u_{t}^{(m)} u_{t+h}^{(m)}\right) & =\frac{1}{4} \mathbb{E}\left(\left[\sum_{j=0}^{m-1} \varepsilon_{t-j}-\sum_{j=0}^{m-1} \varepsilon_{t+m-j}\right]\left[\sum_{j=0}^{m-1} \varepsilon_{t+h-j}-\sum_{j=0}^{m-1} \varepsilon_{t+h+m-j}\right]\right) \\
& = \begin{cases}\frac{2(m-h)-h}{4} \sigma_{\varepsilon}^{2} & \forall h=0 . . m \\
-\frac{(2 m-h)}{4} \sigma_{\varepsilon}^{2} & \forall h=m+1 . .2 m-1 \\
0 & \forall h \geq 2 m .\end{cases}
\end{aligned}
$$

Therefore

$$
r_{u, h}^{(m)}=\frac{\gamma_{h}^{(m)}}{\gamma_{0}^{(m)}}= \begin{cases}1 & h=0 \\ \frac{2 m-3 h}{2 m} & h=1 . . m \\ \frac{m-h}{2 m} & h=m+1 . .2 m-1 \\ 0 & h \geq 2 m .\end{cases}
$$

Proof of Proposition 3 From (23) it can be seen that $u_{t}^{(m)}$ is a linear function of $\varepsilon_{t}$ and is, therefore, also elliptically symmetric (see Hodgson [2006], pp.262-264). When $|\rho|<1, u_{t}$ is also stationary and therefore, by (12), so is $u_{t}^{(m)}$. When $\rho=1$, since $m$ is fixed, $u_{t}^{(m)}$ is stationary by (24) since it is a finite lag function of $\varepsilon_{t}$. Note also that $\mathbb{E}\left[u_{t}^{(m)}\right]=0$. The stated result in (15) then follows directly by the application of (Barnett [1996, pp.20-23]), who generalizes Rice [1944]'s formula to elliptically symmetric processes.

Proof of Proposition 5 We will apply the (Wu [2002], p.642, Theorem 4) central limit theorem for zero-crossings directly to $K_{u, T}^{(m)}(0)$. To do so, we must first show that $u_{t}^{m}$ satisfies the theorem assumptions. For a given $m$, let $a_{j, m} j=0,1, \ldots$ denote the coefficients in the moving average representation of $u_{t}^{m}$, i.e. $u_{t}^{(m)}=\sum_{j=0}^{\infty} a_{j, m} \varepsilon_{t-j}$. Define $A_{k}(\delta) \equiv \sum_{j=k}^{\infty}\left|a_{j}\right|^{\delta}$ as in (Wu [2002], p.642). It is necessary to show that

$$
\sum_{k=1}^{\infty}\left(\frac{A_{k}(\delta)}{k}\right)^{\frac{1}{2}}<\infty
$$

for some $0<\delta \leq 2$ such that $E\left(|\varepsilon|^{\delta}\right)<\infty$. Since, by $(\mathscr{A} .1), E\left(|\varepsilon|^{2}\right)<\infty$, we show this below for $\delta=2$.

When $\rho=1$ or $\rho=0$ it can be seen from (24) or (23), respectively that $u_{t}^{m}$ has a finite order moving average representation, in which case (25) is clearly satisfied, since $A_{k}$ is non-zero for 
only finitely many $k .^{12}$ Together with the condition $(\mathscr{A} .4),(25)$ follows immediately.

When $|\rho|<1$ and $\rho \neq 0, u_{t}^{m}$ can be expressed as in (23). We may ignore the last two terms on the RHS of (23), which again involve finite order moving averages. The infinite moving average in the first term can be re-expressed as

$$
\sum_{j=0}^{\infty} \rho^{j} \varepsilon_{t-m-j}=\rho^{-m}\left[\sum_{i=0}^{\infty} \rho^{i} \varepsilon_{t-i}\right]-\rho^{-m} \sum_{i=0}^{m-1} \rho^{i} \varepsilon_{t-i}
$$

It suffices to verify (25) for the term in brackets, for which

$$
\begin{aligned}
& A_{k}(2)=\sum_{i=k}^{\infty} \rho^{2 i}=\rho^{2 k} \sum_{i=0}^{\infty} \rho^{2 i}=\left(\frac{1}{1-\rho^{2}}\right) \rho^{2 k} \text { and } \\
& \sum_{k=1}^{\infty}\left(\frac{A_{k}(2)}{k}\right)^{\frac{1}{2}} \leq \sum_{k=1}^{\infty}\left(A_{k}(2)\right)^{\frac{1}{2}}=\frac{1}{\left(1-\rho^{2}\right)^{1 / 2}} \sum_{k=1}^{\infty}|\rho|^{k}=\frac{1}{\left(1-\rho^{2}\right)^{1 / 2}(1-|\rho|)}<\infty
\end{aligned}
$$

for $|\rho|<1$. The remaining condition of (Wu [2002], Theorem 4) is satisfied by assumption given (A.6). The stated result then follows by application of $\mathrm{Wu}[2002]$, Theorem 4.

Proof of Proposition 6 For $t<m$ and $t>T-m, u_{t}^{(m)}$ involves the use of the boundary points defined in (2). However, as these involve only $2 m$ points, they can be ignored in the calculation of the limiting variance. Specifically,

$$
\begin{aligned}
\operatorname{Var}\left(K_{u, T}^{(m)}\right)= & T^{-1} \operatorname{Var}\left(\sum_{t=1}^{T-1} \mathbf{1}\left[u_{t}^{(m)} u_{t+1}^{(m)}<0\right]\right)= \\
& T^{-1} \operatorname{Var}\left(\sum_{t=m}^{T-m} \mathbf{1}\left[u_{t}^{(m)} u_{t+1}^{(m)}<0\right]\right)+O_{p}(m / T) .
\end{aligned}
$$

Therefore, since $m=o_{p}(T)$ by assumption,

$$
\begin{aligned}
\lim _{T \rightarrow \infty} \operatorname{Var}\left(K_{u, T}^{(m)}\right)= & \lim _{T \rightarrow \infty} T^{-1} \operatorname{Var}\left(\sum_{t=m}^{T-m} \mathbf{1}\left[u_{t}^{(m)} u_{t+1}^{(m)}<0\right]\right)= \\
& \lim _{T \rightarrow \infty} T^{-1} \sum_{h=-(T-2 m)}^{T-2 m}(T-2 m-|h|) \gamma_{\mathbf{1}[.]}(h)= \\
& \lim _{T \rightarrow \infty} \sum_{h=-(T-2 m)}^{T-2 m}\left(1-\frac{2 m}{T}-\frac{|h|}{T}\right) \gamma_{\mathbf{1}[.]}(h)= \\
& \sum_{h=-\infty}^{\infty} \gamma_{\mathbf{1}[.]}(h) .
\end{aligned}
$$

\footnotetext{
${ }^{12}$ Indeed, as pointed out in by $\mathrm{Wu}[2002]$ in Remark 5, the application of his Theorem 4 is unnecessary in this case because the asymptotic normality follows directly of the $2 m-1$-dependence of $u_{t}^{m}$. As explained in the same remark, the assumption \#\{i: $\left.a_{i} \neq 0\right\}=\infty$ excludes this case only because it already covered by existing results.
} 
The last equality follows because $u_{t}^{m}$ is $2 m-1$-dependent and hence $\mathbf{1}\left[u_{t}^{(m)} u_{t+1}^{(m)}<0\right]$ is $2 m$ dependent, with the implication that $\gamma_{1[\cdot]}(h)=0$ for $h>2 m$ and therefore

$$
\lim _{T \rightarrow \infty} \sum_{h=-(T-2 m)}^{T-2 m}\left(\frac{2 m+|h|}{T}\right) \gamma_{\mathbf{1}[\cdot]}(h)=\lim _{T \rightarrow \infty} \sum_{h=-(2 m)}^{2 m}\left(\frac{2 m+|h|}{T}\right) \gamma_{\mathbf{1}[\cdot]}(h)=0 .
$$

Proof Proof of Corollary 7 (i) follows immediately from the previous results. (ii) holds as a direct consequence of Theorem 1(a) of Andrews [1991] once his Assumptions A and B are established. Define $\theta=E\left[\mathbf{1}\left[u_{t}^{(m)} u_{t+1}^{(m)}<0\right]\right]$ and $v_{t}^{(m)}(\theta)=\mathbf{1}\left[u_{t}^{(m)} u_{t+1}^{(m)}<0\right]-\theta$. To verify Assumption A of Andrews [1991] note first that $v_{t}^{(m)}(\theta)$ is mean zero by construction. It is strictly stationary and therefore fourth order stationary since we assume here that $m$ is fixed $(\mathscr{A} .4)$ and $\rho=1$ it can seen from equation (24) that $u_{t}^{(m)}$ and therefore $v_{t}^{(m)}$ are finite order lag function of $\varepsilon_{t}$, which is strictly stationary as a consequence of Assumption ( $\mathscr{A}$.1). Finally, since $u_{t}^{(m)}$ is $2 m-1$ dependent, $v_{t}^{(m)}(\theta)$ is $2 m$ dependent and therefore satisfies the remaining two summability conditions of Andrews [1991] Assumption A. Andrews [1991]'s Assumption B(i) is satisfied by by the result of Proposition 5. Andrews [1991]'s Assumption B(ii) is trivially satisfied since $V_{t}$ is a (re-centered) indicator function. Since $\partial V_{t}(\theta) / \partial \theta=-1$, Andrews [1991]'s Assumption B(iii) also follows trivially and $\mathrm{B}(\mathrm{iv})$ is assumed in the statement of this corollary.

Proof of Proposition 8 It can be shown that the deterministic component of the transformed series can be expressed in the following way:

$$
\begin{aligned}
D C_{t}= & \left(\alpha_{1}+\beta_{1}\left(t-\lambda_{1} T\right)\right)\left(d_{1, t}-\frac{1}{2} d_{1, t-m}-\frac{1}{2} d_{1, t+m}\right) \\
& +\frac{1}{2} m \beta_{1}\left(d_{1, t-m}-d_{1, t+m}\right)+\ldots \\
& \cdots+\left(\alpha_{p}+\beta_{p}\left(t-\lambda_{p} T\right)\right)\left(d_{p, t}-\frac{1}{2} d_{p, t-m}-\frac{1}{2} d_{p, t+m}\right) \\
& +\frac{1}{2} m \beta_{p}\left(d_{p, t-m}-d_{p, t+m}\right) .
\end{aligned}
$$

Since the break-points dummy variables, $d_{i, t}$ defined in (8), are indicator functions and can only take values of 0 and $1, D C_{t}$ will be equal to zero for values of $t$ satisfying the following conditions:

$$
\begin{gathered}
d_{1, t}-\frac{1}{2} d_{1, t-m}-\frac{1}{2} d_{1, t+m}=0 \\
\vdots \\
d_{p, t}-\frac{1}{2} d_{p, t-m}-\frac{1}{2} d_{p, t+m}=0 .
\end{gathered}
$$

These conditions are equivalent to

$$
\begin{gathered}
d_{1, t-m}=d_{1, t+m} \\
\vdots \\
d_{p, t-m}=d_{p, t+m} .
\end{gathered}
$$

It is apparent that for a process with $p$ breaks that these conditions are satisfied at all points outside $\left[\lambda_{i} T-m, \lambda_{i} T+m\right]$ for $i=0,1,2, \ldots p$. Therefore $x_{t}^{(m)}=u_{t}^{(m)}$ at all but a maximum of 
$p(2 m+1)$ points. Therefore,

$$
\begin{aligned}
\left|K_{T}^{(m)}-K_{u, T}^{(m)}\right| & \leq T^{-1 / 2} \sum_{t=1}^{T-1}\left|\mathbf{1}\left[x_{t}^{(m)} x_{t+1}^{(m)}<0\right]-\mathbf{1}\left[u_{t}^{(m)} u_{t+1}^{(m)}<0\right]\right| \\
& =T^{-1 / 2} \sum_{t=1}^{T-1}\left|\mathbf{1}\left[\left(u_{t}^{(m)}+D C_{t}\right)\left(u_{t+1}^{(m)}+D C_{t+1}\right)<0\right]-\mathbf{1}\left[u_{t}^{(m)} u_{t+1}^{(m)}<0\right]\right| \\
& \leq T^{-1 / 2}(2 m+1) p .
\end{aligned}
$$

\section{References}

Donald W. K. Andrews. Heteroskedasticity and autocorrelation consistent covariance estimation. Econometrica, 59:817-858, 1991.

F. Aparicio, A. Escribano, and A. Sipols. Range unit root (RUR) tests: Robust against nonlinearities, error distributions, structural breaks and outliers. Journal of Time Series Analysis, $27(4): 545-576,2006$.

F. Atkins and M. Chan. Trend breaks and the Fisher hypothesis in Canada and the United States. Applied Economics, 36:1907-1913, 2004.

J. Bai and P. Perron. Estimating and testing linear models with multiple structural changes. Econometrica, 66:47-78, 1998.

A. Banerjee, R.L. Lumsdaine, and J.H. Stock. Recursive and sequential tests of the unit-root and trend-break hypotheses: Theory and international evidence. Journal of Business and Economic Statistics, 10(2):271-287, 1992.

J.T. Barnett. Zero-Crossing Rates of Some Non-Gaussian Processes with Applications to Detection and Estimation. PhD thesis, University of Maryland, 1996.

W.B. Brown and D.J. Hodgson. Semiparametric efficiency bounds in dynamic non-linear systems under elliptical symmetry. Econometrics Journal, 10:35-48, 2007.

P. Burridge and E. Guerre. The limit distribution of level crossings of a random walk. Econometric Theory, 12:705-723, 1996.

A. Cowles and H. Jones. Some a posteriori probabilities in stock market action. Econometrica, $5(3): 280-294,1937$.

S. Csörgó and J. Mielniczuk. The empirical process of a short-range dependent stationary sequence under Gaussian subordination. Probability Theory and Related Fields, 104:15-25, 1996.

G. Elliott, T. Rothenberg, and J. Stock. Efficient test for an autoregressive unit root. Econometrica, 64:813-836, 1996.

Martin D. D. Evans and Karen K. Lewis. Do expected shifts in inflation affect estimates of the long-run Fisher relation? The Journal of Finance, 50(1):225-253, 1995.

Kai-Tai Fang, Samuel Kotz, and Kai Wang Ng. Symmetric multivariate and related distributions. Number 36 in Monographs on statistics and applied probability. Chapman \& Hall, London [u.a.], 1990. ISBN 0412314304. 
I. Fisher. The Theory of Interest. The Macmillan Co., New York, 1930.

A. Garcia and A. Sanso. A generalization of the Burridge-Guerre nonparametric unit root test. Econometric Theory, 22:756-761, 2006.

H. Ho and T. Hsing. Limit theorems for functionals of moving averages. The Annals of Probability, 25(4):1636-1669, 1997.

H. Ho and T. Sun. A central limit theorem for non-instantaneous filters of a stationary gaussian process. Journal of Multivariate Analysis, 22:144-155, 1987.

H.C. Ho. On functionals of linear processes with estimated parameters. Statistica Sinica, 12: 1171-1190, 2002.

D. J. Hodgson and K. Vorkink. Efficient estimation of conditional asset pricing models. Journal of Business and Economic Statistics, 21:269-283, 2003.

D. J. Hodgson, O. Linton, and K. Vorkink. Testing the capital asset pricing model efficiently under elliptical symmetry: A semiparametric approach. Journal of Applied Econometrics, 17: 617-639, 2002.

D.J. Hodgson. On efficient, robust, and adaptive estimation in cointegrated models. In D. Corbae, S.N. Durlauf, and B.E. Hansen, editors, Econometric Theory and Practice: Frontiers of Analysis and Applied Research, chapter 9, pages 241-265. Cambridge University Press, 2006.

T. Hsing. On the asymptotic distributions of partial sums of functionals of infinite-variance moving averages. The Annals of Probability, 27(3):1579-1599, 1999.

T. Hsing. Linear processes, long-range dependence and asymptotic expansions. Statistical Inference for Stochastic Processes, 3:19-29, 2000.

G. Kapetanios. Unit-root testing against the alternative hypothesis of up to $\mathrm{m}$ structural breaks. Journal of Time Series Analysis, 26(1):123-133, 2005.

M. G. Kendall. The analysis of economic time-series-part i: Prices. Journal of the Royal Statistical Society. Series A, General., 116(1):11-34, 1953.

D. Kim and P. Perron. Unit root tests allowing for a break in the trend function at an unknown time under both the null and alternative hypotheses. Journal of Econometrics, 148(1):1 - 13, 2009.

A. Lo and C. MacKinlay. Stock market prices do not follow random walks: Evidence from a simple specification test. The Review of Financial Studies, 1(1):41-66, 1988.

R. Lumsdaine and D. Papell. Multiple trend breaks and the unit-root hypothesis. The Review of Economics and Statistics, 79(2):212-218, 1997.

Frederic S. Mishkin. Is the Fisher effect for real? : A reexamination of the relationship between inflation and interest rates. Journal of Monetary Economics, 30(2):195 - 215, 1992.

C. Nelson and C. Plosser. Trends and random walks in macroeconomic time-series: some evidence and implications. Journal of Monetary Economics, 10(2):139-162, 1982.

Whitney K. Newey and Kenneth D. West. A simple positive semi-definite, heteroskedasticity and autocorrelation consistent covariance matrix. Econometrica, 55:703-708, 1987.

H. Ohara. A unit root test with multiple trend breaks: A theory and an application to US and Japanese macroeconomic time-series. The Japanese Economic Review, 50(3):266-290, 1999. 
Joel Owen and Ramon Rabinovitch. On the class of elliptical distributions and their applications to the theory of portfolio choice. The Journal of Finance, 38(3):745-752, 1983.

P. Dorian Owen. Cointegration analysis of the Fisher hypothesis: the role of the real rate and the Fisher identity. Applied Financial Economics, 3(1):21 - 26, 1993.

H. Park and W. Fuller. Alternative estimators and unit root tests for the autoregressive process. Journal of Time Series Analysis, 16:415-429, 1995.

P. Perron. The Great Crash, the oil price shock, and the unit root hypothesis. Econometrica, 57:1361-1401, 1989.

P. Perron. Cointegration for the applied economist., chapter Trend, Unit Root and Structural change in macroeconomic time series., pages 113-146. The Macmillan Press, 1994.

P. Perron and T. Vogelsang. Nonstationary time series and level shifts with an application to purchasing power parity. Journal of Business and Economic Statistics, 10:301-320, 1992.

S. Rice. The mathematical analysis of random noise. Bell Systems Technical Journal, 23:282-332, 1944.

M. Sinn and K. Keller. Covariances of zero crossing in mean-zero Gaussian processes. Theory of Probability and Its Applications, (forthcoming), 2010.

Minoru Tanaka and Kunio Shimizu. Discrete and continuous expectation formulae for levelcrossings, upcrossings and excursions of ellipsoidal processes. Statistics \& Probability Letters, 52(3):225-232, April 2001.

M.S. Taqqu. Convergence of integrated processes of arbitrary hermite rank. Zeitschrift für Wahrscheinlichkeitstheorie und verwandte Gebiete, 50:53-83, 1979.

T. Vogelsang. On testing for a unit root in the presence of additive outliers. Working papers, Cornell - Department of Economics, 1994.

W.B. Wu. Central limit theorems for functionals of linear processes and their applications. Statistica Sinica, 12:635-649, 2002.

W.B. Wu. Additive functionals of infinite-variance moving averages. Statistica Sinica, 13:12591267, 2003.

Z. Zhao and W.B. Wu. Asymptotic theory for curve-crossing analysis. Stochastic Processes and their Applications, 117:862-877, 2007.

E. Zivot and D.W.K. Andrews. Further evidence on the great crash, the oil-price shock, and the unit-root hypothesis. Journal of Business and Economic Statistics, 10(2):251-270, 1992. 